\title{
Controlling dipolar exchange interactions in a dense three-dimensional array of large-spin fermions
}

\author{
A. Patscheider $\odot,{ }^{1}$ B. Zhu $\odot,{ }^{2,3,4}$ L. Chomaz $\odot,{ }^{1}$ D. Petter $\odot,{ }^{1}$ S. Baier $\odot,{ }^{1}$ A.-M. Rey $\odot,{ }_{,}^{4}$ F. Ferlaino $\odot,{ }^{1,5}$ and M. J. Mark $\odot^{1,5, *}$ \\ ${ }^{1}$ Institut für Experimentalphysik, Universität Innsbruck, 6020 Innsbruck, Austria \\ ${ }^{2}$ ITAMP, Harvard-Smithsonian Center for Astrophysics, Cambridge, Massachusetts 02138, USA \\ ${ }^{3}$ Department of Physics, Harvard University, Cambridge, Massachusetts 02138, USA \\ ${ }^{4}$ JILA, NIST, and Department of Physics, University of Colorado, 440 UCB, Boulder, Colorado 80309, USA \\ ${ }^{5}$ Institut für Quantenoptik und Quanteninformation, Österreichische Akademie der Wissenschaften, 6020 Innsbruck, Austria
}

(Received 27 September 2019; revised manuscript received 19 December 2019; accepted 13 March 2020; published 17 April 2020)

\begin{abstract}
Dipolar interactions are ubiquitous in nature and rule the behavior of a broad range of systems spanning from energy transfer in biological systems to quantum magnetism. Here we study magnetization-conserving dipolar induced spin-exchange dynamics in dense arrays of fermionic erbium atoms confined in a deep three-dimensional lattice. Harnessing the special atomic properties of erbium, we demonstrate control over the spin dynamics by tuning the dipole orientation and changing the initial spin state within the large 20-spin hyperfine manifold. Furthermore, we demonstrate the capability to quickly turn on and off the dipolar exchange dynamics via optical control. The experimental observations are in excellent quantitative agreement with numerical calculations based on discrete phase-space methods, which capture entanglement and beyond-mean-field effects. Our experiment sets the stage for future explorations of rich magnetic behaviors in long-range interacting dipoles, including exotic phases of matter and applications for quantum information processing.
\end{abstract}

DOI: 10.1103/PhysRevResearch.2.023050

\section{INTRODUCTION}

Spin lattice models of localized magnetic moments (spins), which interact with one another via exchange interactions, are paradigmatic examples of strongly correlated many-body quantum systems. Their implementation in clean, isolated, and fully controllable lattice confined ultracold atoms opens a path for a new generation of synthetic quantum magnets, featuring highly entangled states, especially when driven out of equilibrium, with broad applications ranging from precision sensing and navigation to quantum simulation and quantum information processing [1,2]. However, the extremely small energy scales associated with the nearest-neighbor spin interactions in lattice-confined atoms with dominant contact interactions have made the observation of quantum magnetic behaviors extremely challenging $[3,4]$. On the contrary, even under frozen motional conditions, dipolar gases, featuring long-range and anisotropic interactions, offer the opportunity to bring ultracold systems several steps ahead toward the ambitious attempt of modeling and understanding quantum magnetism. Great advances in studying quantum magnetism have been achieved using arrays of Rydberg atoms [5-8], trapped ions [9-11], polar molecules [12,13], and spin-3

\footnotetext{
*Corresponding author: manfred.mark@uibk.ac.at
}

Published by the American Physical Society under the terms of the Creative Commons Attribution 4.0 International license. Further distribution of this work must maintain attribution to the author(s) and the published article's title, journal citation, and DOI. bosonic chromium atoms $[14,15]$. Most of these studies so far have been limited to spin-1/2 mesoscopic arrays of at most a few hundred particles or to macroscopic but dilute $(<0.1$ filling fractions) samples of molecules in lattices or were contaminated by the effects of double occupancies in the system. Only very recently, experiments using bosonic chromium gas have revealed the power of magnetic atoms toward the understanding of quantum magnetism [16].

Here we report on a substantial leap forward in the control and understanding of atomic dipolar platforms for simulating quantum magnetism. Relying on the atomic properties of erbium, we realize a quantum simulator of the long-range $X X Z$ Heisenberg model in a dense array of fermionic magnetic atoms with unique control knobs. Our platform roots on the special atomic properties of ${ }^{167} \mathrm{Er}$. The erbium ground state bears large angular momentum quantum numbers with $I=7 / 2$ for the nuclear spin and $J=6$ for the electronic angular momentum, resulting in a $F=19 / 2$ hyperfine manifold. These large numbers bring in several important consequences and provide novel control capabilities. First, they give access to a fully addressable landscape of $2 F+1=20$ internal levels, as depicted in Fig. 1(a). Second, they are responsible for the large magnetic moment in erbium, leading to a strong dipolar coupling between atoms in neighboring lattice sites. Such coupling can be up to 49 times larger than the ones felt by $F=1 / 2$ alkali atoms and about $40 \%$ larger than the one in chromium [17]. Finally, the non- $S$ character of the ground state leads to comparatively large tensorial light shifts [18], which enables spin-dependent optical manipulation in addition to the quadratic Zeeman effect [19].

In our work, any of the 20 internal spin states available in erbium can be selected to initialize our simulator in a quantum 

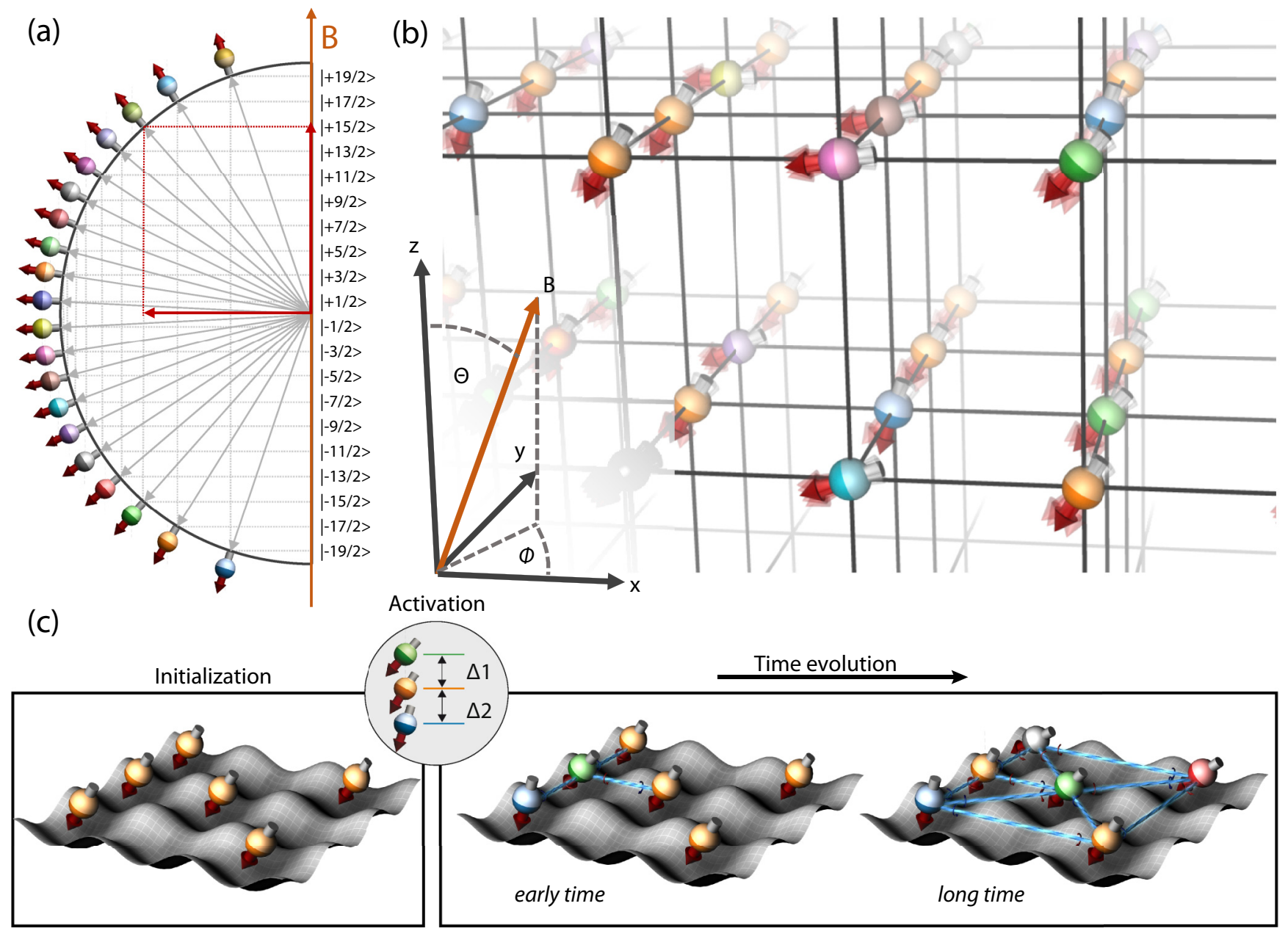

FIG. 1. (a) Illustration of the total spin space of a single ${ }^{167} \mathrm{Er}$ atom in the lowest hyperfine level $|F=19 / 2\rangle$ with all $20 m_{F}$ states. The angle of the symbols indicates the orientation of the total spin $|\mathbf{F}|=\sqrt{F(F+1)}$ in relation to the quantization axis. (b) Sketch of the experimental system, an anisotropic three-dimensional lattice structure filled with fermionic ${ }^{167} \mathrm{Er}$ with a quantization axis tunable by the angles $(\Theta, \phi)$ of the external magnetic field $B$. (c) Illustration of the experimental sequence (from left to right): The system is initialized by preparing all atoms in one starting state, here $|-17 / 2\rangle$. We activate the spin dynamics by changing the magnetic field to set $\bar{\delta}=0$. Early-time dynamics are happening mainly among nearest-neighbor atoms. Subsequently, interactions between atoms at larger distances are involved in the dynamics.

spin Fock state. After activating spin-exchange processes, arising from the large magnetic dipole interaction of erbium, we study the spreading of the spin population, characterize the effective strength of the dipolar coupling, and observe evidence of beyond-nearest-neighbor couplings. We benchmark our simulator with an advanced theoretical approach, which takes quantum entanglement and spatial inhomogeneities into account [20]; see Appendix H. Moreover, we show that the spin-exchange dynamics can be fully controlled via optical light fields on timescales much faster than typical interaction times. The reported demonstration of these new control knobs, some without equivalence in alkali and chromium atoms, constitutes an important step toward a fully controllable quantum simulator, e.g., for the realization of synthetic dimension [21-23] or as qudits for quantum computation [24-26].

\section{THEORETICAL DESCRIPTION}

The $X X Z$ Heisenberg model that rules the magnetizationconserving spin dynamics of our system can be conveniently written using spin-19/2 dimensionless angular momentum operators $\hat{\mathbf{F}}_{i}=\left\{\hat{F}_{i}^{x}, \hat{F}_{i}^{y}, \hat{F}_{i}^{z}\right\}$, acting on site $i$ and satisfying the commutation relation $\left[\hat{F}_{i}^{x}, \hat{F}_{i}^{y}\right]=i \hat{F}_{i}^{z}$. We use the eigenbasis of $\hat{F}^{z}$ denoted as $\left|m_{F}\right\rangle$ with $0 \leqslant\left|m_{F}\right| \leqslant F \quad[27,28]$ (see Appendix F):

$$
\begin{aligned}
\hat{H}= & \frac{1}{2} \sum_{i, j \neq i} V_{i, j}\left[\hat{F}_{i}^{z} \hat{F}_{j}^{z}-\frac{1}{4}\left(\hat{F}_{i}^{+} \hat{F}_{j}^{-}+\hat{F}_{i}^{-} \hat{F}_{j}^{+}\right)\right] \\
& +\sum_{i} \delta_{i}\left(\hat{F}_{i}^{z}\right)^{2} .
\end{aligned}
$$

The coupling constants $V_{i, j}=V_{d d} \frac{1-3 \cos ^{2}\left(\theta_{i, j}\right)}{r_{i j}^{3}}$ describe the direct dipole-dipole interactions (DDI), which have longrange character and thus couple beyond nearest neighbors. The dipolar coupling strength between two dipoles located at $\vec{r}_{i}$ and $\vec{r}_{j}$ depends on their relative distance $r_{i j}=\left|\vec{r}_{i}-\vec{r}_{j}\right|$ and on their orientation, described by the angle $\theta_{i, j}$ between the dipolar axis, set by the external magnetic field, and the 

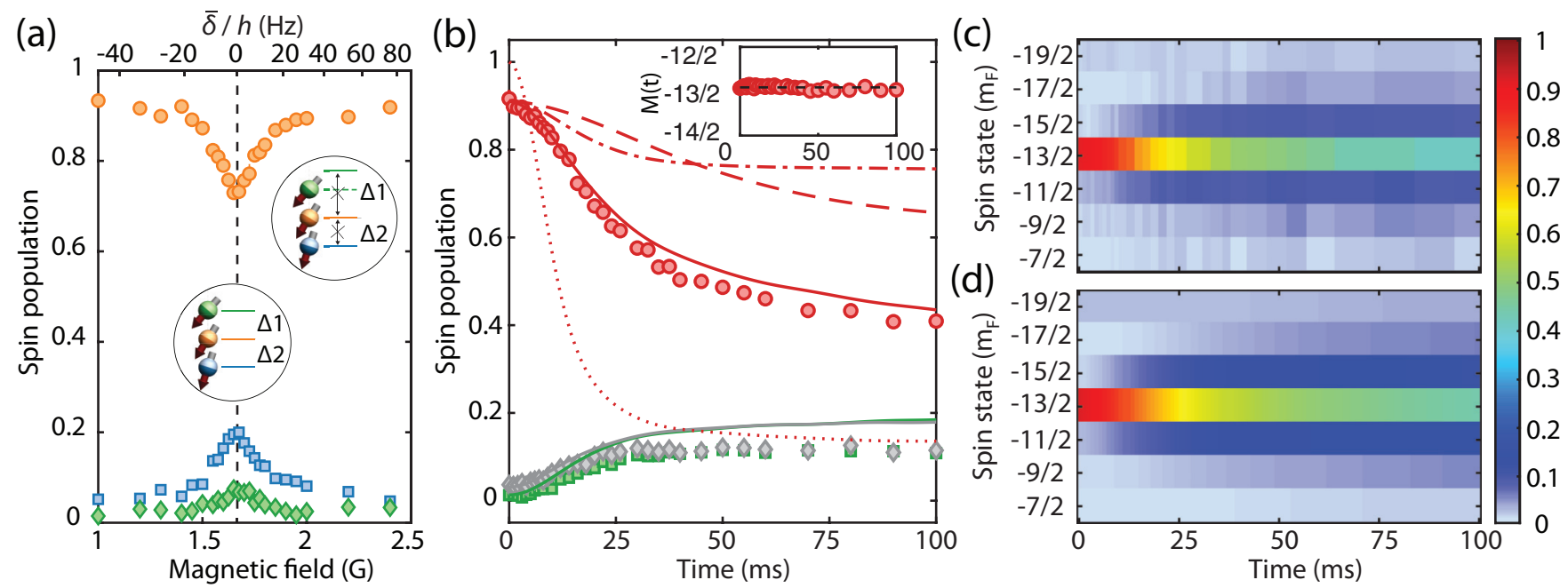

FIG. 2. (a) Measured spin populations in states $m_{F}=|-17 / 2\rangle$ (circles) and $m_{F} \pm 1$ (diamonds and squares) after $50 \mathrm{~ms}$ hold time as a function of the magnetic field with $\Theta=0^{\circ}$. A Gaussian fit (not shown) to the data provides a resonant magnetic field value of $\approx 1.67 \mathrm{G}$. The top axis shows the mean total detuning $\bar{\delta}$ from the resonance condition. (b) Measured spin population in states $m_{F}=|-13 / 2\rangle$ (circles) and $m_{F} \pm 1$ (diamonds and squares) as a function of the hold time after quenching onto the spin-exchange resonance with $\Theta=0^{\circ}$. The dashed line shows the simple mean-field result, the dashed-dotted line gives the NNI-GDTWA result, the solid lines represent the full-GDTWA result, and the dotted line shows the full-GDTWA for the ideal case of a lattice with unit filling. The inset shows the total magnetization $M(t)$. [(c) and (d)] Spin diffusion with initial state $m_{F}^{0}=|-13 / 2\rangle$ plotting the population of states from $m_{F}-3$ to $m_{F}+3$ as a function of the hold time, for the experiment (c) and the full-GDTWA model (d), with the same initial conditions as (b). Data points consist of a minimum of four individual realizations and error bars denote the standard error of the mean.

interparticle axis; see Fig. 1(b). Here $V_{d d} \approx \frac{\mu_{0} g_{F}^{2} \mu_{B}^{2}}{4 \pi}$ denotes the dipolar coupling strength, with $g_{F} \approx 0.735$ for ${ }^{167} \mathrm{Er}, \mu_{0}$ the magnetic permeability of vacuum, and $\mu_{B}$ the Bohr magneton. The $\hat{F}_{i}^{z} \hat{F}_{j}^{z}$ terms in the Hamiltonian account for the diagonal part of the interactions while the $\hat{F}_{i}^{+} \hat{F}_{j}^{-}+\hat{F}_{i}^{-} \hat{F}_{j}^{+}$terms describe dipolar exchange processes. The second sum denotes the single-particle quadratic term $\delta_{i}\left(\hat{F}_{i}^{z}\right)^{2}$ with $\delta_{i}=\delta_{i}^{Z}+\delta_{i}^{T}$, accounting for the quadratic Zeeman effect $\propto \delta_{i}^{Z}$ and tensorial light shifts $\propto \delta_{i}^{T}$. These two contributions can be independently controlled in our experiment.

The quadratic Zeeman shift allows us to selectively prepare all atoms in one target state of the spin manifold; see Appendix B. The tensorial light shift can compete or cooperate with the quadratic Zeeman shift and can be used as an additional control knob to activate/deactivate the exchange processes. Note that, for all measurements, a large linear Zeeman shift is always present, but since it does not influence the spin-conserving dynamics, it is omitted in Eq. (1).

\section{EXPERIMENTAL SEQUENCE}

In the experiment, we first load a spin-polarized quantum degenerate Fermi gas of $\approx 10^{4}$ Er atoms into a deep threedimensional (3D) optical lattice, following the scheme of Ref. [29]. The cuboid lattice geometry with lattice constants $\left(d_{x}, d_{y}, d_{z}\right)=(271(2), 266(1), 542(4)) \mathrm{nm}$ results in weakly coupled 2D planes, with typical tunneling rates of $\sim 10 \mathrm{~Hz}$ inside the planes and $\sim \mathrm{mHz}$ between them; see Appendix E. The external magnetic field orientation, setting the quantization axis as well as the dipolar coupling strengths, is defined by the polar angles $\Theta$ and $\phi$ in the laboratory frame; see Fig. 1(b). The fermionic statistics of the atoms enables us to prepare a dense band insulator with at most one atom per lattice site. Additionally, Pauli exclusion blocks tunneling as well as superexchange processes on the initial timescales, helping in realizing a frozen condition, as required for a clean implementation of the $X X Z$ Heisenberg model; see Appendix $J$. This is an advantage of fermionic atoms as compared to bosonic systems, which typically require filtering protocols to remove doublons and higher lattice depths to avoid tunneling [16].

Our experimental sequence to study the spin dynamics is illustrated in Fig. 1(c). In particular, we prepare the system into the targeted spin Fock state, with a near-unity population of the $m_{F}^{0}$ level, by using the lattice-protection protocol demonstrated in Ref. [29]. At the end of the preparation, the majority of atoms are in the desired $m_{F}^{0}(>80 \%)$ at $B \approx 4 \mathrm{G}$. We note that atom losses during the spin preparation stage reduces the filling factor to about $60 \%$ of the initial one; see Appendix D. We then activate the spin dynamics by quenching the magnetic field to a value for which $\bar{\delta}=\sum_{i} \delta_{i}=0$, providing a resonance condition for the magnetization-conserving spinexchange processes; see Fig. 2(a) with $m_{F}^{0}=|-17 / 2\rangle$. After a desired time of evolution, we stop the dynamics by rapidly increasing the magnetic field, leaving the resonance condition. We finally extract the atom number in each spin state via a spin-resolved band-mapping technique and derive the relative state populations by normalization to the initial total atom number. Note that, throughout all our experiments, within our detection resolution, we do not observe any population in higher bands.

We now probe the evolution of the spin-state population as a function of the hold time on resonance. We observe a redistribution of the population from the initial state to multiple neighboring states in $m_{F}$ space, as for exemple shown for an initial state of $|-13 / 2\rangle$ in Figs. 2(b) and 2(c). The 
dynamics preserves the total magnetization; see the inset of Fig. 2(b). We observe similar behavior independently of the initialized $m_{F}^{0}$ states. The spin transfer happens sequentially. At short times it is dominated by the transfer to states directly coupled by the dipolar exchange Hamiltonian, i.e., those ones which differ by plus/minus one unit of angular momentum $\left(\Delta m_{F}= \pm 1\right)$. At longer times, subsequent processes transfer atoms to states with $\left|\Delta m_{F}\right| \geqslant 2$; see Figs. 2(c) and 2(d).

\section{COMPARISON TO GDTWA}

To benchmark our quantum simulator, we use a semiclassical phase-space sampling method, the so-called generalized discrete truncated Wigner approximation (GDTWA) [16,20,30-32]; see Appendix G. The method accounts for quantum correlation in the many-body dynamics and is adapted to tackle the complex dynamics of a large-spin system in a regime where exact diagonalization techniques are impossible to implement with current computers. The GDTWA calculations take into account actual experimental parameters such as spatial inhomogeneites, density distribution after the lattice loading, initial spin distribution, and effective lattice filling, including the loss during the spin preparation protocol; see Appendices D and H. Figure 2(b) shows the experimental dynamics together with the GDTWA simulations. Although the model does not include corrections due to losses and tunneling during the dynamics, it successfully captures the behavior of our dense system not only at short time but also at long time, where the population dynamics slows down and starts to reach an equilibrium. A similar level of agreement between experiment and theory is shown in Figs. 2(c) and 2(d) where we directly compare the spreading of the spin population as a function of time.

Our choice of using a quantum Fock state instead of a spin coherent state [16] as initial state combined with our possibility to cancel the quadratic energy shift (i.e., set $\bar{\delta}=0$ ) allows us to directly reveal the important role of quantum effects in the observed spin dynamics. This can be shown both at the quantitative and qualitative levels by contrasting the GDTWA simulation with a mean-field calculation. Indeed, the mean-field calculation fails in capturing the system behavior. It predicts a too-slow population dynamics for nonperfect spin-state initialization, as in the experiments shown in Fig. 2(b), and no dynamics for the ideal case where all atoms are prepared in the same internal state. To emphasize the beyond-nearest-neighbor effects, we also compare the experiment with a numerical simulation that only includes nearestneighbor interactions (NNI-GDTWA). Here we again observe a very slow spin evolution, which largely deviates from the measurements. The agreement of the full GDTWA predictions with our experimental observations points to the long-range many-body nature of the underlying time evolution. Our theory calculations also support the build-up of entanglement during the observed time evolution. To illustrate the quantum spin dynamics under ideal conditions, in Fig. 2(b), we also show the results for a system with unity filling and perfect initialization in state $m_{F}^{0}=|-13 / 2\rangle$. In this case a speedup of the dynamics due to the increased effective interaction strength is visible, quickly approaching an equilibrium state.

\section{CONTROL OF THE INTERACTION STRENGTH}

Different spin configurations feature distinct effective interaction strengths, which also depend on the orientation of the dipoles with respect to the lattice. We demonstrate our ability to control this interaction, which governs the rate of population exchange, by the choice of the initial $m_{F}^{0}$ state and the orientation of the external magnetic field. This capability provides us with two tuning knobs to manipulate dipolar exchange interactions in our quantum simulator. Figures 3(a)-3(f) plot the dynamics of the populations for three neighboring spin states after the quench, starting from different initial spin states. Solid lines show the results of the full-GDTWA calculations. For each initial $m_{F}^{0}$, we find a remarkable agreement between theory and experiment. We observe a strong speedup for states with large spin projections perpendicular to the quantization axis, as is expected from the expectation value of $\hat{F}_{i}^{+} \hat{F}_{j}^{-}$, which gives a prefactor $\gamma\left(m_{F}^{0}\right)=$ $\sqrt{F(F+1)-m_{F}^{0}\left(m_{F}^{0}+1\right)} \sqrt{F(F+1)-m_{F}^{0}\left(m_{F}^{0}-1\right)}$. The initial dynamics can be well described by a perturbative expansion up to the second order (see Appendix I), resulting in the analytic expression for the normalized population $n_{m_{F}}(t)$ of the initial state:

$$
n_{m_{F}^{0}}(t)=n_{m_{F}^{0}}(0)\left[1-n_{m_{F}^{0}}(0) \frac{V_{\mathrm{eff}}^{2}}{\hbar^{2}} t^{2}\right] .
$$

Here $V_{\text {eff }}^{2} \equiv \frac{\gamma^{2}\left(m_{F}^{0}\right)}{8 N} \sum_{i, j \neq i} V_{i j}^{2}$ is the overall effective interaction strength summed over $N$ atoms and $n_{m_{F}^{0}}(0)$ denotes the purity of the initial state preparation. For a quantitative analysis of the early-time spin evolution, we compare the theoretically calculated $V_{\text {eff }}$ from the initial atomic distribution used in the GDTWA model with the one extracted from a fit of Eq. (2) to the experimental data. Here we consider the data up to $t<0.5 \frac{\hbar}{V_{\text {eff }}}$ estimated using the theoretically calculated $V_{\text {eff }}$ [33]. Figure $3(\mathrm{~g})$ plots both the theoretical and experimental $V_{\text {eff }}$ as a function of the initial $m_{F}^{0}$ and highlights once more their quantitative agreement. The interaction parameter $V_{\text {eff }}$ can also be used to rescale the time axis. As shown in Fig. 3(h), all data sets now collapse onto each other for $\frac{t V_{\text {eff }}}{\hbar}<0.5$, revealing the invariant character of the short-time dynamics under the internal state initialization. At longer timescales, the theory shows that the timetraces start to deviate from each others and saturate to different values, indicating that thermal-like states are on reach. In the experiment, we observe a similar behavior but here the saturation value might also be affected by losses and residual tunneling.

Because of the anisotropic character of the DDI, the strength of the dipolar exchange interaction can be controlled by changing the angle $\Theta$; see Fig. 1(b). As shown in Fig. 4(a) for $|-17 / 2\rangle$, the observed evolution speed of the spin populations strongly depends on $\Theta$, changing by about a factor of 2 between $\Theta=40^{\circ}$ and $80^{\circ}$. The GDTWA results show a very good quantitative agreement with the experiment. We repeat the above measurements for different values of $\Theta$ and we extract $V_{\text {eff }}$; Fig. 4(b). It is worthwhile to mention that, while the dipolar interactions can be completely switched off at a given angle in a $1 \mathrm{D}$ chain, in a $3 \mathrm{D}$ system the situation is more complicated. However, as expected by geometrical arguments, we observe that the overall exchange strength 

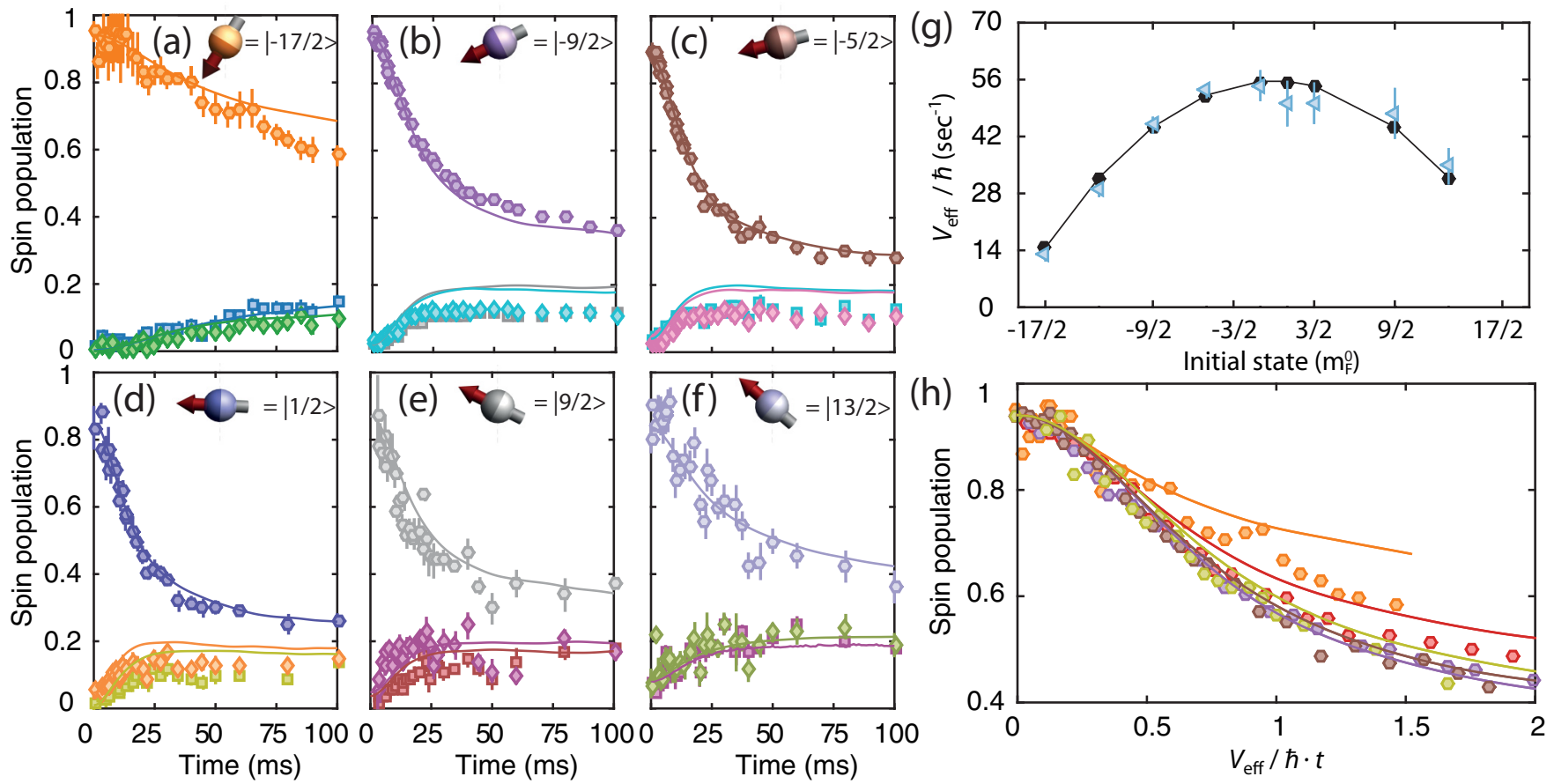

FIG. 3. [(a)-(f)] Dynamic evolution of the initial states $|-17 / 2\rangle$ (a), $|-9 / 2\rangle$ (b), $|-5 / 2\rangle$ (c), $|1 / 2\rangle$ (d), $|9 / 2\rangle$ (e), and $|13 / 2\rangle$ (f) and of the corresponding neighboring states $m_{F} \pm 1$ together with the full-GDTWA results (solid lines) for $\Theta=0^{\circ}$. (g) Extracted $V_{\text {eff }}$ as a function of the initial state $m_{F}^{0}$ from a fit to the experimental data (cyan triangles) and numerically computed from the initial spin distribution (black circles). Error bars denote the $68 \%$ confidence interval of the fits. (h) All datasets with $m_{F}^{0}<0$ used in (g) together with the corresponding full-GDTWA results (solid lines) plotted in units of the rescaled time $\tau=V_{\text {eff }} / \hbar \cdot t$. Note that all experiment and theory data are plotted for times $t \leqslant 100 \mathrm{~ms}$ of (a)-(f). To account for the different preparation fidelity, the populations of the initial states are shifted to 1 by adding a constant offset. For clarity error bars are omitted here.

becomes minimal for a specific dipole orientation $\left(\Theta_{c} \approx 35^{\circ}\right.$, $\left.\phi_{c}=45^{\circ}\right)$. We compare our measured $V_{\text {eff }}$ with the ones calculated from the initial spin distribution, which is a good quantity to describe the early-time dynamics. Theory and experiment show a similar trend, in particular reaching a minimum at about $\Theta_{c}$. Note that the simple analytic formula [Eq. (2)], used for fitting the data, deviates from the actual evolution at longer times. This leads to a small down-shift of the experimental values; see Appendix I. Our study ultimately demonstrates the ability to tune the lattice spin model through the magnetic field orientation. This paves the way to the simulation of various spin-lattice models [34].

\section{OPTICAL CONTROL}

Finally, we demonstrate fast optical control of the spin dynamics relying on the remarkably large tensorial light shift of erbium compared to alkali atoms. As shown in Fig. 4(c), we can almost fully suppress the spin-exchange dynamics by suddenly switching on a homogeneous light field after an initial evolution time on resonance. Therefore, the tensorial light shift, inducing a detuning from the resonance condition (see inset), allows a full spatial and temporal control over the exchange processes as light fields can be easily shaped in space and time, in stark contrast to magnetic fields. For example, the light power can be changed orders of magnitude faster than the typical interaction times and can address even single lattice sites in quantum gas microscopes [35-37].
By exploiting the rich energy spectrum of lanthanides like erbium, narrow optical transitions [38] allow an even more refined manipulation of the spin manifold like, e.g., blocking specific spin-exchange channels or additional Floquet engineering. This capabilities can be an excellent resource for quantum information processing, e.g., we could use dipolar exchange processes to efficiently prepare highly entangled states between different parts of a quantum system and then store the quantum information at longer times by turning the interactions off.

\section{CONCLUSION AND OUTLOOK}

In our work, we explore the dipolar exchange dynamics and benchmark our simulator with an advanced theoretical model, which takes quantum entanglement and spatial inhomogeneities into account. In particular, we initialize the system into a desired spin Fock state and activate the spin dynamics, while the motional degree of freedom mainly remains frozen. We study the spreading of the spin population with different choices of the macroscopically populated initial Zeeman level as a function of both the specific initial spin state and the dipole orientation. We demonstrate that the spin dynamics at short evolution time follows a scaling that is invariant under internal state initialization and that is set by the effective strength of the dipolar coupling. On the contrary, at longer times, the many-body dynamics is affected by the accessible spin space and the long-range character 

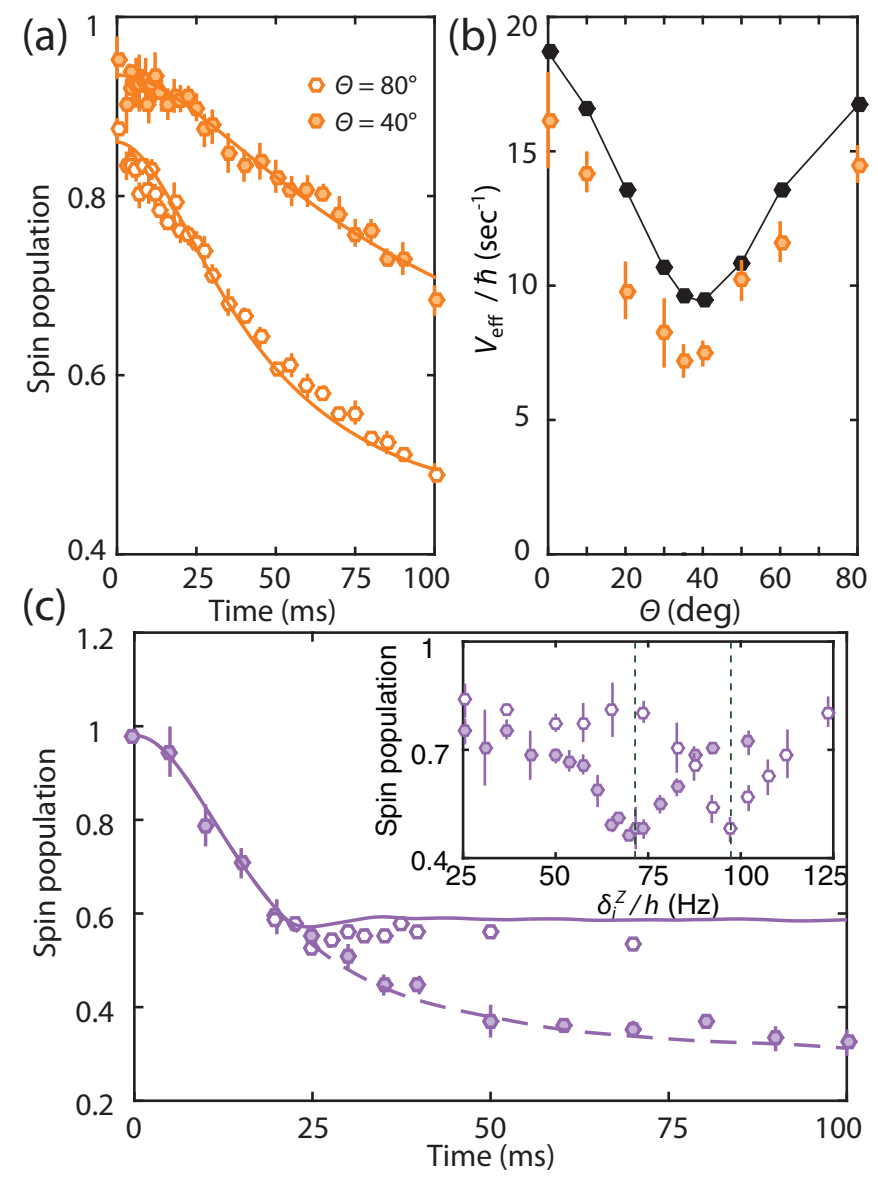

FIG. 4. (a) Exemplary measurements of the time evolution for the starting spin state $|-17 / 2\rangle$ for $\Theta=40^{\circ}, 80^{\circ}$. Solid lines show the full-GDTWA results. (b) Extracted $V_{\text {eff }}$ as a function of $\Theta$ from a fit to the experimental data (orange circles) and numerically computed from the initial spin distribution (black circles). Error bars denote the $68 \%$ confidence interval of the fits. (c) Time evolution of the initial state $|-9 / 2\rangle$ at $\bar{\delta}=0$ and $\Theta=0^{\circ}$ without (filled circles) and with (open circles) switching on an additional light field after $20 \mathrm{~ms}$ of evolution. Solid (dashed) lines are the corresponding full-GDTWA calculations. The inset shows the population of the initial spin state after 50 ms evolution time as a function of the quadratic Zeeman shift without (filled circles) and with (open circles) the additional light field. Determining the centers of the resonances via a fit yields an absolute shift of the resonance condition by $h \times 27(1) \mathrm{Hz}$ between both conditions.

of dipolar interactions beyond nearest neighbors. We further demonstrate temporal control of the exchange dynamics using off resonant laser light.

The excellent agreement between the experiment and the theory not only verifies our quantum simulator but also sets the stage toward its use for the study of new regimes intractable to theory. For example, by operating in a shallow lattice where motion is involved, the dynamics is no longer described by a spin model but by a high spin Fermi-Hubbard model with long-range interactions. Alternatively by treating the internal hyperfine levels as a synthetic dimension [21,22] while adding Raman transitions to couple them, one could engineer nontrivial synthetic gauge field models even when tunneling is only allowed in one direction. Moreover, the demonstrated control over the initial state population of arbitrary hyperfine levels, our capability to tune the strength of the direct dipolar exchange coupling via the magnetic field angle, and the possibility of the dynamical and spatial control of the hyperfine manifold via tensorial light shifts make our system a potential resource for quantum information processing with a qudit-type multilevel encoding using the 20 different interconnected hyperfine levels [24-26].

\section{ACKNOWLEDGMENTS}

We thank J. Schachenmayer for fruitful discussions and Arghavan Safavi-Naini for helping us understanding the loading into the lattice. We thank J. H. Becher and G. Natale for their help in the experimental measurements and for fruitful discussions. We also thank Rahul Nandkishore and Itamar Kimchi for reviewing the manuscript. The Innsbruck group is supported through an ERC Consolidator Grant (RARE, Grant No. 681432), an EC Horizon 2020 FET Proactive project (RySQ, Grant No. 640378), and a DFG/FWF Forschergruppe (FOR 2247/PI2790). L.C. is supported though an EC Horizon 2020 Marie Curie Project (DipPhase, Grant No. 706809). A.M.R is supported by the ARO single investigator Award No. W911NF-19-1-0210, DARPA-DRINQs, the NSF PHY1820885, JILA-NSF PFC-173400 grants, and by NIST. B.Z. is supported by the NSF through a grant to ITAMP.

\section{APPENDIX A: EXPERIMENTAL SETUP AND LATTICE LOADING}

In our experiment we start with a degenerate Fermi gas of about $2.4 \times 10^{4}{ }^{167} \mathrm{Er}$ atoms in the lowest spin state $\left|F=19 / 2, m_{F}=-19 / 2\right\rangle=|-19 / 2\rangle$ and a temperature of $T \approx 0.3 T_{F}$ [29,39]. The atoms are confined in a crossed optical dipole trap (ODT) and the trap frequencies are $\left(v_{\perp}, v_{\|}, v_{z}\right)=(63(1), 36(2), 137(1)) \mathrm{Hz}$, where $v_{\perp}\left(v_{\|}\right)$are the trap frequencies perpendicular to (along) the horizontal ODT and $v_{z}$ is measured along the vertical direction defined by gravity. We load the atomic sample adiabatically into a $3 \mathrm{D}$ lattice that is created by two retroreflected laser beams at $532 \mathrm{~nm}$ in the $x-y$ plane and one retroreflected laser beam at $1064 \mathrm{~nm}$ nearly along the $z$ direction, defined by gravity and orthogonal to the $x-y$ plane. Note that due to a small angle of about $11(2)^{\circ}$ between the vertical lattice beam and the $z$ axis we obtain a 3D lattice, slightly deviating from an ideal situation of a rectangular unit cell and our parallelepipedic cell has the unit lattice distances of $d_{x}=271(2) \mathrm{nm}, d_{y}=266(1) \mathrm{nm}$, and $d_{z}=542(2) \mathrm{nm}$. The lattice geometry is similar to the one used in our previous works [29,40]. We ramp up the lattice beams in $150 \mathrm{~ms}$ to their final power and switch off the ODT subsequently in $10 \mathrm{~ms}$ and wait for $500 \mathrm{~ms}$ to eliminate residual atoms in higher bands [29]. For our typical lattice depths used in the measurements reported here of $\left(s_{x}, s_{y}, s_{z}\right)=(20,20,80)$, where $s_{i}$ with $i \in x, y, z$ is given in the respective recoil energy $\mathrm{E}_{R, i}$ with $E_{\mathrm{R} ; x, y}=h \times 4.2 \mathrm{kHz}$ and $E_{\mathrm{R} ; z}=h \times 1.05 \mathrm{kHz}$, the atoms can be considered pinned on single lattice sites with low tunneling rates $J_{x, y}=h \times 10.5 \mathrm{~Hz}$ and $J_{z}=h \times 1 \mathrm{mHz}$. 


\section{APPENDIX B: STATE PREPARATION AND DETECTION EFFICIENCY}

To prepare the atoms in the desired spin state, after loading them into the lattice, we use a technique based on a rapid-adiabatic passage (RAP). During the full preparation procedure, the optical lattice operates as a protection shield to avoid atom loss and heating due to the large density of Feshbach resonances [29]. To reach a reliable preparation with high fidelity it is necessary that the change in the energy difference between subsequent neighboring spin states is sufficiently large. Therefore, we ramp the magnetic field in $40 \mathrm{~ms}$ to $40.6 \mathrm{G}$ to get a large-enough differential quadratic Zeeman shift, which is on the order of $\approx h \times 40 \mathrm{kHz}$. After the magnetic field ramp we wait for $80 \mathrm{~ms}$ to allow the latter to stabilize before performing the RAP procedure. The follow up RAP protocol depends on the target state. For example, to transfer the atoms from $|-19 / 2\rangle$ into the $|-7 / 2\rangle$ state, we apply a radio-frequency (RF) pulse at $41.31 \mathrm{MHz}$ and reduce the magnetic field with a linear ramp, e.g., by $500 \mathrm{mG}$ in $42 \mathrm{~ms}$. The variation of the magnetic field is analogous to the more conventional scheme where the frequency of the RF is varied [see Fig. 5(a)]. For the preparation of higher (lower) spin states we perform a larger (smaller) reduction of the magnetic field on a longer (shorter) timescale. After the RAP ramp we switch off the RF field and ramp the magnetic field in $10 \mathrm{~ms}$ to $B=3.99 \mathrm{G}$. Here we wait again for $100 \mathrm{~ms}$ to allow the magnetic field to stabilize. During the ramp up and down to $40 \mathrm{G}$ of the magnetic field we loose $25(2) \%$ of the atoms. We attribute this loss mainly to the dense Feshbach spectra that we are crossing when ramping the magnetic field. The exact loss mechanism has not been yet identified, constituting a topic of interest for latter investigation. At $3.99 \mathrm{G}$, before switching on the spin dynamics, about $1.7 \times 10^{4}$ atoms remain in the lattice. The losses affect the lattice filling at which the spin dynamics occur. Our simulations account for this initially reduced filling; see Appendix $\mathrm{H}$.

Additionally to the losses due to the magnetic field ramps, we also observe losses caused by the RAP itself. To quantify the preparation efficiency, i.e., the loss of atoms due to the spin preparation via RAP as a function of the target $m_{F}$ state, we perform additional measurements where we either do not perform a RAP or where we add an inverse RAP to transfer all atoms back into the $|-19 / 2\rangle$ state. By comparing the atom numbers from measurements without RAP and with double-RAP and assuming that the loss process is symmetric, we derive the preparation efficiency as plotted in Fig. 5(b). We also account for the difference in the counting efficiency of the individual spin states, which arises from different effective scattering cross sections for the imaging light. Here we compare the measured atom number in a target $m_{F}$ state to the expected atom number taking into account the previously determined preparation efficiency as discussed above and the atom number without RAP; see Fig. 5(c).

The counting and preparation efficiencies are determined for the $|-17 / 2\rangle,|-15 / 2\rangle,|-9 / 2\rangle$, and $|9 / 2\rangle$ states and interpolated assuming a linear dependency of these efficiencies on $m_{F}$ [see Figs. 5(b) and 5(c)]. We estimate the preparation efficiency of the respective $m_{F}$ state to be $0.86(1)-0.008(1) \times m_{F}$. We attribute the lower preparation
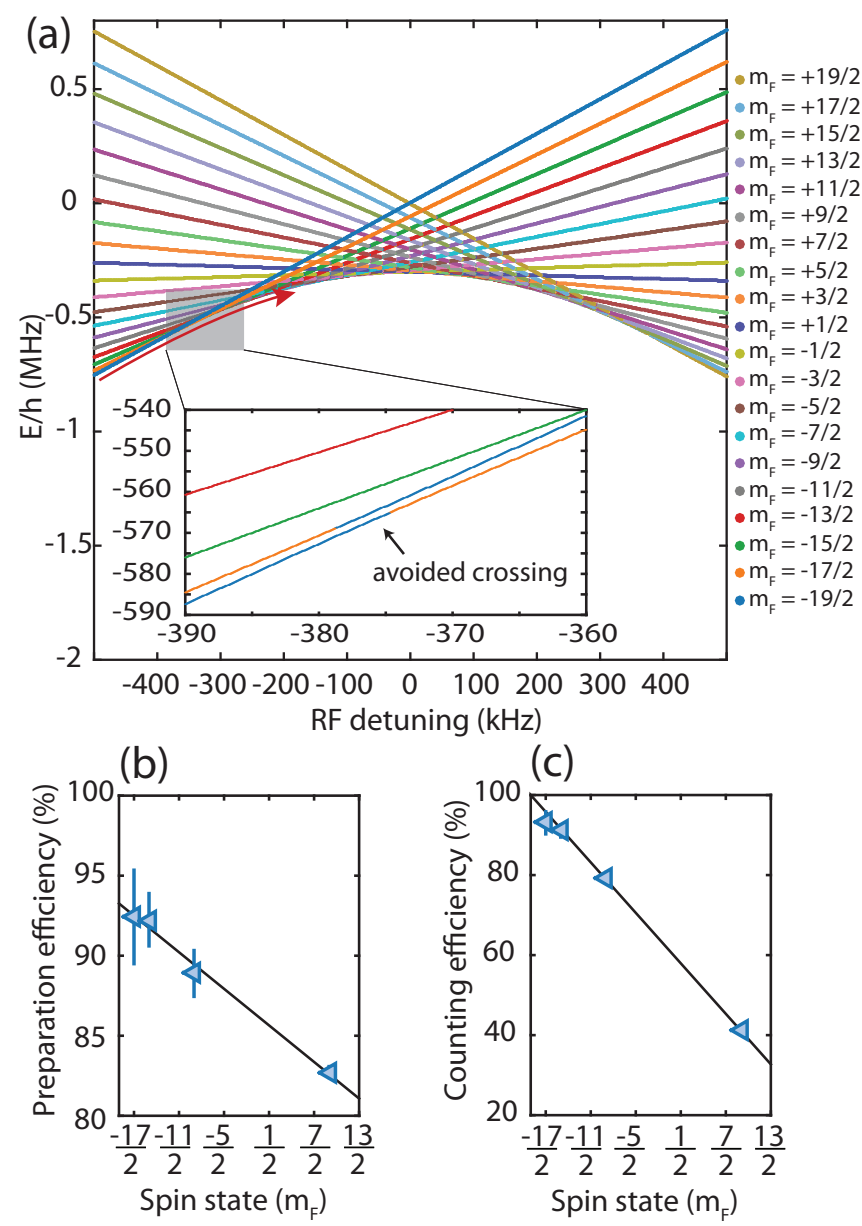

FIG. 5. (a) Energy levels of the ground-state hyperfine manifold in the dressed-state picture in dependence of the detuning between the applied just RF and the atomic resonance condition for the $|-1 / 2\rangle$ to the $|1 / 2\rangle$ hyperfine levels. The solid red arrow exemplary shows the RAP for preparation of atoms into the $m_{F}^{0}=|-7 / 2\rangle$ state. The insets show a zoom of one avoided crossing. [(b) and (c)] Spinpreparation and atom-counting efficiency measured for $|-17 / 2\rangle$, $|-15 / 2\rangle,|-9 / 2\rangle$, and $|9 / 2\rangle$. The obtained values are interpolated linearly assuming a linear dependence on the $m_{F}^{0}$ state.

efficiency for higher spin states to the larger number of avoided crossing between spin states that come into play during the RAP procedure. Overall, we expect that the lattice filling over the whole sample, taking into account the losses due to magnetic field ramping and spin state preparation, reduces from close to unity down to a value between 0.6 and 0.7; see also Tables I and II.

\section{APPENDIX C: QUENCH PROTOCOL AND DETECTION SEQUENCE}

In our experiment we exploit both the light and the magnetic shifts of the energies of each spin state to reach a resonant condition where the energy difference between neighboring spin states is canceled and therefore spin changing dynamics preserving the total magnetization become energetically allowed. In particular, we exploit the tensorial light 
TABLE I. Percentage of lost atoms and extracted, effective filling fraction $v$ for different $\left|m_{F}\right\rangle$ states at $t=t_{\mathrm{fit}}$ and $t=100 \mathrm{~ms}$.

\begin{tabular}{|c|c|c|c|c|c|c|}
\hline$m_{F}^{0}$ & $t_{\text {fit }}(\mathrm{ms})$ & $N_{\text {loss }}\left(t_{\text {fit }}\right)(\%)$ & $v(0)$ & $v\left(t_{\mathrm{fit}}\right)$ & $N_{\text {loss }}(100 \mathrm{~ms})(\%)$ & $v(100 \mathrm{~ms})$ \\
\hline$-\frac{17}{2}$ & 34.2 & 1.8 & 0.7 & 0.68 & 5.3 & 0.66 \\
\hline$-\frac{13}{2}$ & 15.7 & 7.2 & 0.69 & 0.64 & 19.6 & 0.55 \\
\hline$-\frac{9}{2}$ & 11.3 & 8.7 & 0.67 & 0.62 & 25.1 & 0.51 \\
\hline$-\frac{5}{2}$ & 9.6 & 13.7 & 0.66 & 0.58 & 27.7 & 0.48 \\
\hline$-\frac{1}{2}$ & 9.0 & 12.2 & 0.65 & 0.57 & 36.1 & 0.42 \\
\hline$\frac{1}{2}$ & 9.0 & 13.5 & 0.65 & 0.56 & 36.7 & 0.41 \\
\hline$\frac{3}{2}$ & 9.2 & 9.2 & 0.64 & 0.58 & 34.0 & 0.43 \\
\hline$\frac{9}{2}$ & 11.3 & 6.7 & 0.63 & 0.59 & 23.1 & 0.49 \\
\hline$\frac{13}{2}$ & 15.7 & 4.9 & 0.62 & 0.59 & 21.9 & 0.48 \\
\hline
\end{tabular}

shift of the spin states energies [18]

$$
U_{t}=\frac{3 m_{F}^{2}-F(F+1)}{F(2 F-1)} \frac{3 \cos ^{2} \theta_{p}-1}{2} \alpha_{t}(\omega),
$$

present in atomic erbium to initialize the dynamic evolution of the spin population. The tensorial light shift depends quadratically on the $m_{F}$ state as well as on the angle $\theta_{p}$ between the magnetic field axis and the axis of polarization of the light. Here $\alpha_{t}$ refers to the tensorial polarizability coefficient and $\omega$ to the light frequency. After the preparation of the respective spin state we start all our measurements at $B=3.99 \mathrm{G}$, pointing in the $z$ direction. However, to reach the resonance condition we use two slightly different quench protocols for the measurement sets with fixed $\Theta=0^{\circ}$ for the different initial spin state and for the sets of measurements where $\left|m_{F}\right\rangle=|-17 / 2\rangle$ and $\Theta \in\left(0^{\circ}, 80^{\circ}\right)$. The measurement sequences differ on the one hand by the way we jump on resonance to initialize the spin dynamics and on the other hand by shining in an additional laser beam of wavelength $1064 \mathrm{~nm}$ and power of $7 \mathrm{~W}$. This additional light is necessary because changing $\Theta$ reduces simultaneously $\theta_{p}$, resulting in a smaller tensorial light shift and therefore in a shift of the resonance position to lower magnetic field values. For large $\Theta$ the light shift of the lattice beams is smaller and therefore the resonance is very close to $0 \mathrm{G}$ which we want to avoid to prevent spin relaxation processes. For the sets of measurements where we keep $\Theta=0^{\circ}$ but vary the initial $m_{F}^{0}$ state we quench the magnetic field directly after the preparation, from $3.99 \mathrm{G}$ to resonance. In contrast we use a different approach for the measurements where $\Theta$ is varied. After the preparation we ramp in $10 \mathrm{~ms}$ the additional laser beam to $7 \mathrm{~W}$. Due to the reduced speed of our magnetic field coils in the $x$ and $y$ directions we first rotate the magnetic field such that the transverse components $B_{x}$ and $B_{y}$ are already at their target values while keeping an additional offset of $2 \mathrm{G}$ in the $z$ direction. The quench to resonance is then done using only the coils for the magnetic field in the $z$ direction. The additional offset field of $2 \mathrm{G}$ is large enough to suppress dynamics. We measure the evolution of the magnetic field by performing RF spectroscopy and find that for both quench procedures the magnetic field evolves exponentially toward its quench value with $1 / e$ decay times of 1.4 and $1.2 \mathrm{~ms}$, respectively. After holding on resonance for a certain time we quench the magnetic field back to $3.99 \mathrm{G}$ and we rotate the latter back to $\Theta=0^{\circ}$. After a waiting time of $50 \mathrm{~ms}$ we perform a band-mapping measurement combined with a Stern-Gerlach technique, i.e., we ramp the lattice down in $1 \mathrm{~ms}$ and apply a magnetic field gradient that is large enough to separate the individual spin states after a time of flight (TOF) of $15 \mathrm{~ms}$. This allows us to image the first Brillouin zone for the different spin states. During TOF the magnetic field is rotated toward the imaging axis. We typically record the population of the initially prepared $\left|m_{F}\right\rangle$, of its four neighbors, and of $|-19 / 2\rangle$ by summing the 2D atomic density over a region of interest. Figure 6 shows examples of the imaging of different spin states for the cases of a nonadjusted RAP as well as for the preparation of the atoms in $|-9 / 2\rangle,|3 / 2\rangle$, and $|5 / 2\rangle$. In the case of $|3 / 2\rangle$ residual atoms in $|-19 / 2\rangle,|-17 / 2\rangle$, and $|5 / 2\rangle$ are visible due to a nonperfect preparation.

TABLE II. Percentage of lost atoms and extracted effective filling fraction $v$ for different $\Theta$ at $t=t_{\text {fit }}$ and $t=100 \mathrm{~ms}$.

\begin{tabular}{|c|c|c|c|c|c|c|}
\hline$\Theta(\operatorname{deg})$ & $t_{\text {fit }}(\mathrm{ms})$ & $N_{\text {loss }}\left(t_{\text {fit }}\right)(\%)$ & $v(0)$ & $v\left(t_{\text {fit }}\right)$ & $N_{\text {loss }}(100 \mathrm{~ms})(\%)$ & $v(100 \mathrm{~ms})$ \\
\hline 0 & 26.8 & 13.8 & 0.7 & 0.60 & 30.1 & 0.49 \\
\hline 10 & 30.1 & 11.9 & 0.7 & 0.61 & 25.6 & 0.52 \\
\hline 20 & 36.7 & 6.9 & 0.7 & 0.65 & 18.2 & 0.57 \\
\hline 30 & 47.0 & 8.6 & 0.7 & 0.64 & 17.8 & 0.57 \\
\hline 35 & 52.0 & 6.7 & 0.7 & 0.65 & 11.4 & 0.62 \\
\hline 40 & 53.2 & 7.2 & 0.7 & 0.65 & 13.2 & 0.60 \\
\hline 50 & 46.1 & 12 & 0.7 & 0.61 & 17.2 & 0.58 \\
\hline 60 & 37.0 & 8.9 & 0.7 & 0.63 & 19.2 & 0.56 \\
\hline 80 & 30.0 & 10.4 & 0.7 & 0.62 & 19.3 & 0.56 \\
\hline
\end{tabular}




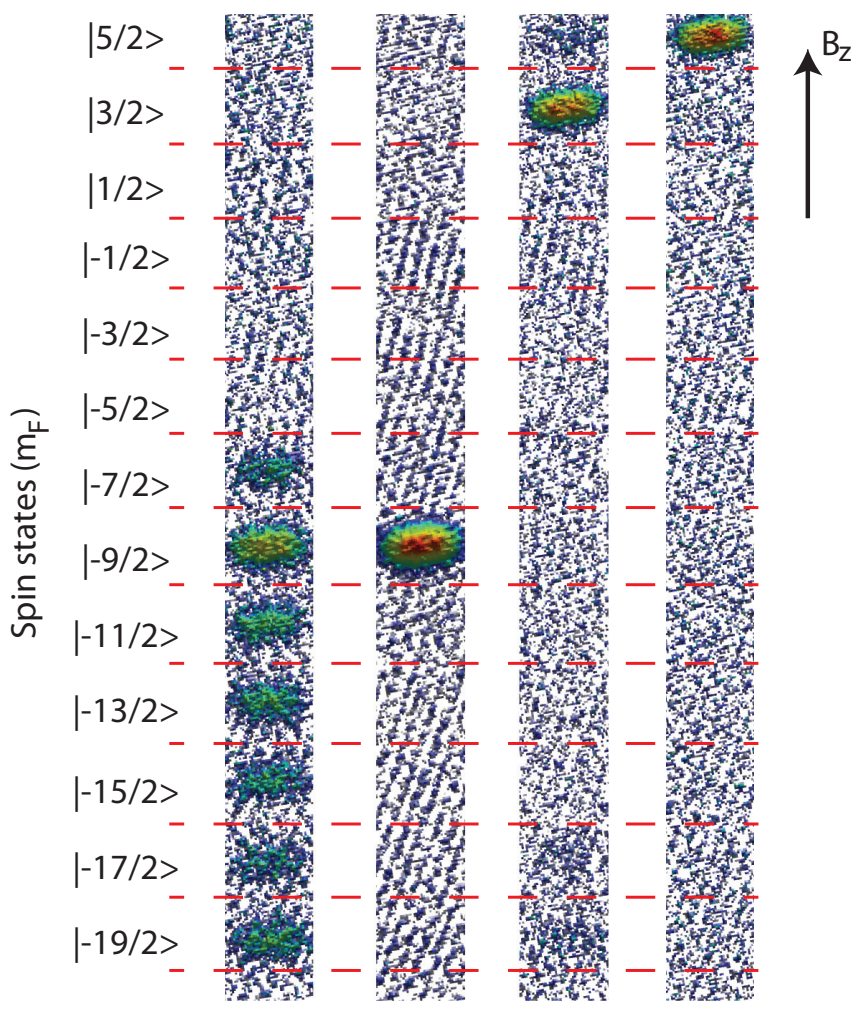

FIG. 6. Absorption images for a nonadjusted RAP and for the preparation of $|-9 / 2\rangle,|3 / 2\rangle$, and $|5 / 2\rangle$. Whereas for the $|-9 / 2\rangle$ and $|5 / 2\rangle$ case no residual atoms in other spin states are visible, for the $|3 / 2\rangle$ case we observe a small amount of residual atoms in other spin states due to a nonperfect preparation.

\section{APPENDIX D: LIFETIME AND LOSSES IN THE LATTICE}

Off-resonance, i.e., at a magnetic field of $B=3.99 \mathrm{G}$, we measure the lifetime of the prepared spin state to be on the order of a few seconds, being slightly shorter for higher spin states. Note that here we do not observe any population growing in the neighboring spin states. Differently, for the measurements on resonance, we observe a faster loss happening on the timescale of the first 20-30 ms followed by loss at lower speed for the remaining atoms. We fit an exponential decay to extract the atoms loss and change in filling over the timescale that we use to extract $V_{\text {eff }}, t_{\text {fit }}$, (see Appendix I) as well as over the full $100 \mathrm{~ms}$ of the dynamics reported in the main text (Figs. 2 and 3). Table I gives the corresponding numbers for the sets of data for the different initial $m_{F}^{0}$ states. During the fitting timescale we observe atoms loss on the order of $5-10 \%$. This atom loss can be converted into a change of the effective filling of the lattice compared to the state obtained after the lattice loading giving a minimum filling of $v=0.56$ for the $m_{F}^{0}=|1 / 2\rangle$ case. For longer timescales larger losses in the range between 10 and $35 \%$ are observed. In general, we note that the amount of loss depends on the initial $m_{F}^{0}$ state, resulting larger for the central $\left|m_{F}\right\rangle^{0}$ states. Similar numbers are obtained for the sets of measurement where we vary $\Theta$ (see Table II). The exact mechanism leading to these losses is not yet understood and will be the topic of future studies. Thanks to their limited importance over the early-time dynamics, we here compare our results to theoreti- cal prediction without losses; see Incorporating Experimental Conditions in Numerical Simulation. A proper description of the long-time dynamics will certainly require to account and understand such effects.

\section{APPENDIX E: EXPERIMENTAL UNCERTAINTIES AND INHOMOGENEITIES}

Ideally, all atoms in the sample experience the same linear and quadratic Zeeman shift and the same quadratic light shift. However, in the experiment inhomogeneities from the magnetic field and light intensities lead to a spatial dependence of those shifts. An upper bound of the variation of Zeeman shifts can be deduced from RF-spectroscopy measurements done with bosonic erbium. From the width of the RF resonance $(\approx 500 \mathrm{~Hz})$ and the size of the cloud $(\approx 15 \mu \mathrm{m})$ we estimate a maximum magnetic field gradient of $\leqslant 230 \mathrm{mG} / \mathrm{cm}$, assuming the gradient as main broadening mechanism for the resonance width, neglecting magnetic field noise and Fourier broadening. This translates into a differential linear Zeeman shift of $\leqslant h \times 6 \mathrm{~Hz}$ between adjacent lattice sites in the horizontal $x-y$ plane and $\leqslant h \times 12 \mathrm{~Hz}$ between adjacent planes along the $z$ direction. Together with the magnetic field values used in the spin dynamic experiments, the variation of the quadratic Zeeman shift is negligible compared to other inhomogeneities $(\leqslant h \times 0.1 \mathrm{~Hz})$. The inhomogeneity of the quadratic light shifts can be estimated by considering the shape of the lattice light beams [Gaussian beams with waists of about $\left.\left(w_{x}, w_{y}, w_{z}\right)=(160,160,300) \mu \mathrm{m}\right]$ and the resonance condition of the magnetic field, translated into a quadratic Zeeman shift of $h \times 71(1) \mathrm{Hz}$. These considerations can be used to obtain an estimation for a site-dependent light shift compared to the center of the atomic sample. If we take a possible displacement of the atoms by $\leqslant 10 \mu \mathrm{m}$ in all directions, from the center of the lattice to the center of the beams, into account, then we can estimate an upper bound for the light shift of $\delta_{i}^{T} \leqslant h \times 2 \mathrm{~Hz}$ at 20 lattice sites away from the center along the $y$ direction.

\section{APPENDIX F: SPIN HAMILTONIAN}

The experiment operates in a deep lattice regime, where tunneling is suppressed. At the achieved initial conditions, the ${ }^{167} \mathrm{Er}$ atoms are restricted to occupy the lowest lattice band, and Fermi statistics prevents more than one atom per lattice site. In the presence of a magnetic field strong enough to generate Zeeman splittings larger than nearest-neighbor dipolar interactions, only those processes that conserve the total magnetization are energetically allowed [15]. Under these considerations, the dynamics is described by the following secular Hamiltonian:

$$
\begin{aligned}
\hat{H}= & \sum_{i} \delta_{i}\left(\hat{F}_{i}^{z}\right)^{2}+\sum_{i} B_{i} \hat{F}_{i}^{z} \\
& +\frac{1}{2} \sum_{i, j \neq i} V_{i, j}\left[\hat{F}_{i}^{z} \hat{F}_{j}^{z}-\frac{1}{4}\left(\hat{F}_{i}^{+} \hat{F}_{j}^{-}+\text {H.c. }\right)\right] .
\end{aligned}
$$

Here the operators $F_{i}^{z, \pm}$ are spin 19/2 angular momentum operators acting on lattice site $i$. The first two terms account for the site-dependent quadratic and linear shifts, respectively, 
where $\delta_{i}$ includes both Zeeman terms and tensorial light shifts as discussed in the main text. $B_{i}=B+\Delta B_{i}$ denotes the linear Zeeman shift at site $i$. While the constant and uniform contribution, $B$, commutes with all other terms, thus can be rotated out, the spatially varying contribution, $\Delta B_{i}$, is relatively small in the experiment but still is accounted for in the theory calculations. The last term is the long-range dipolar interaction between atoms in different sites, with

$$
V_{\mathbf{i}, \mathbf{j}} \equiv V_{d d} d_{y}^{3} \frac{1-3 \cos ^{2} \theta_{i, j}}{\left|\mathbf{r}_{i}-\mathbf{r}_{j}\right|^{3}}
$$

where $\theta_{i j}$ is the angle between the dipolar orientation set by an external magnetic field and the interparticle spacing $\mathbf{r}_{i}-\mathbf{r}_{j}$. $V_{d d} \approx \frac{\mu_{0} g_{F}^{2} \mu_{B}^{2}}{4 \pi d_{y}^{3}}$ corresponds to the interaction strength between two atoms, $i$ and $j$, separated by the smallest lattice constant $\left|\mathbf{r}_{i}-\mathbf{r}_{j}\right|=d_{y}=266 \mathrm{~nm}$ and forming an angle $\theta_{i, j}=\pi / 2$ with the quantization axis. Here $g_{F} \approx 0.735$ is the Lande $g$ factor for Er atoms, $\mu_{0}$ is the magnetic permeability of vacuum, and $\mu_{B}$ is the Bohr magneton. We compute $V_{d d}$ from

$V_{d d}=\frac{\mu_{0}\left(\mu_{B} g_{F}\right)^{2}}{4 \pi} \int d^{3} \mathbf{r} d^{3} \mathbf{r}^{\prime} \frac{1-3 \cos ^{2} \theta_{r r^{\prime}}}{\left|\mathbf{r}-\mathbf{r}^{\prime}\right|^{3}}\left|\phi_{i}(\mathbf{r})\right|^{2}\left|\phi_{j}\left(\mathbf{r}^{\prime}\right)\right|^{2}$,

where $\phi_{i}(r)$ denotes the lowest band Wannier function centered at lattice site $i$. For the experimental lattice depths $\left(s_{x}, s_{y}, s_{z}\right)=(20,20,80)$ in units of the corresponding recoil energies, $V_{d d}$ is estimated to be $h \times 0.336 \mathrm{~Hz}$.

\section{APPENDIX G: THE GDTWA METHOD}

To account for quantum many-body effects during the dynamics generated by long-range dipolar interactions in these complex macroscopic spin $F=19 / 2$ 3D lattice array, we apply the so-called GDTWA, first introduced in Ref. [16]. The underlying idea of the method is to supplement the mean-field dynamics of a spin $F$ system with appropriate sampling over the initial conditions in order to quantitatively account for the build-up of quantum correlations. For a spin-F atom $i$ with $\mathcal{N}=2 F+1$ spin states, its density matrix $\hat{\rho}_{i}$ consists of $\mathcal{D}=\mathcal{N} \times \mathcal{N}$ elements. Correspondingly, we can define $\mathcal{D}$ Hermitian operators, $\Lambda_{\mu}^{i}$, with $\mu=1, \ldots, D$, using the generalized Gell-Mann matrices (GGM) and the identity matrix [41]:

$$
\Lambda_{\mu=1, \ldots, \mathcal{N}(\mathcal{N}-1) / 2}^{i}=\frac{1}{\sqrt{2}}(|\beta\rangle\langle\alpha|+\text { H.c. }),
$$

for $\alpha>\beta, 1 \leqslant \alpha, \beta \leqslant \mathcal{N}$,

$$
\Lambda_{\mu=\mathcal{N}(\mathcal{N}-1) / 2+1, \ldots, \mathcal{N}(\mathcal{N}-1)}^{i}=\frac{1}{\sqrt{2} i}(|\beta\rangle\langle\alpha|-\text { H.c. })
$$

for $\alpha>\beta, 1 \leqslant \alpha, \beta \leqslant \mathcal{N}$,

$$
\begin{aligned}
\Lambda_{\mu}^{i} & =\mathcal{N}(\mathcal{N}-1)+1, \ldots, \mathcal{N}^{2}-1 \\
& =\frac{1}{\sqrt{\alpha(\alpha+1)}}\left(\sum_{\beta=1}^{\alpha}|\beta\rangle\langle\beta|-\alpha| \alpha+1\rangle\langle\alpha+1|\right),
\end{aligned}
$$

for $1 \leqslant \alpha<\mathcal{N}$

$$
\Lambda_{\mathcal{D}}^{i}=\sqrt{\frac{1}{\mathcal{N}}} \mathbb{I}
$$

With these operators, the local density matrix $\hat{\rho}_{i}$, as well as any operator $\hat{O}^{i}$ of local observables can be represented as

$$
\begin{aligned}
\hat{O}^{i} & =\sum_{\mu} c_{\mu}^{i} \Lambda_{\mu}^{i}, \quad \text { with } \\
c_{\mu}^{i} & =\operatorname{Tr}\left[\Lambda_{\mu}^{i} \hat{O}^{i}\right],
\end{aligned}
$$

and $\mu=1,2, \ldots, \mathcal{D}$. This allows expressing both one-body and two-body Hamiltonians in the form $\hat{H}_{i}=\sum_{\mu} c_{\mu}^{i} \Lambda_{\mu}^{i}$ and $\hat{H}_{i j}=\sum_{\mu, \nu} c_{\mu \nu}^{i j} \Lambda_{\mu}^{i} \Lambda_{\nu}^{j}$. The Heisenberg equations of motion for $\Lambda_{\mu}^{i}$ can be written as

$$
\begin{aligned}
i \hbar \frac{d \Lambda_{\mu}^{i}}{d t} & =\left[\Lambda_{\mu}^{i}, \hat{H}\right] \\
& =\sum_{\mu} c_{\nu}^{i}\left[\Lambda_{\mu}^{i}, \Lambda_{\nu}^{i}\right]+\sum_{\sigma, j, \nu} c_{\sigma, \nu}^{i j}\left[\Lambda_{\mu}^{i}, \Lambda_{\sigma}^{i}\right] \Lambda_{\nu}^{j} .
\end{aligned}
$$

In the experiment, the initial state is a product state of singleatom density matrices $\hat{\rho}(t=0)=\prod \hat{\rho}^{i}(t=0)$. If we adopt a factorization $\left\langle\Lambda_{\mu}^{i} \Lambda_{\nu}^{j} \cdots \Lambda_{\sigma}^{k}\right\rangle=\left\langle\Lambda_{\mu}^{i}\right\rangle\left\langle\Lambda_{\nu}^{j}\right\rangle \cdots\left\langle\Lambda_{\sigma}^{k}\right\rangle$ for any nonequal $i, j, \ldots, k$ (i.e., each operator acts on a different atom) and arbitrary $\mu, v, \sigma$, then Eq. (G7) becomes a closed set of nonlinear equations for $\lambda_{\mu}^{i}=\left\langle\Lambda_{\mu}^{i}\right\rangle$. Within a mean-field treatment, the initial condition is fixed by $\lambda_{\mu}^{i}(t=0)=\operatorname{Tr}\left[\Lambda_{\mu}^{i} \hat{\rho}(t=0)\right]$, which determines the ensuing dynamics from Eq. (G7). This treatment neglects any correlations between atoms. In the GDTWA method, the initial value of $\lambda_{\mu}^{i}$ is instead sampled from a probability distribution in phase space, with statistical average $\overline{\lambda_{\mu}^{i}(0)}=\operatorname{Tr}\left[\Lambda_{\mu}^{i} \hat{\rho}(t=0)\right]$. Specifically, each $\Lambda_{\mu}^{i}$ can be decomposed via its eigenvalues and eigenvectors as $\Lambda_{\mu}^{i}=\sum_{a_{\mu}^{i}} a_{\mu}^{i}\left|a_{\mu}^{i}\right\rangle\left\langle a_{\mu}^{i}\right|$. We take $a_{\mu}^{i}$ as the allowed values of $\Lambda_{\mu}^{i}$ in phase space, and then for an initial state $\hat{\rho}^{i}(t=0)$, the probability distribution is $p\left(a_{\mu}^{i}\right)=\operatorname{Tr}\left[\hat{\rho}^{i}(t=0)\left|a_{\mu}^{i}\right\rangle\left\langle a_{\mu}^{i}\right|\right]$. From Eq. (G7), each sampled initial configuration for the $N$ atom array, $\left\{a_{\mu}\right\}=\left\{a_{\mu_{1}}^{i 1}, a_{\mu_{2}}^{i 2}, \ldots, a_{\mu_{N}}^{i N}\right\}$, leads to a trajectory of $\Lambda_{\mu}^{i}$, which we denote as $\lambda_{\mu,\left\{a_{\mu}\right\}}^{i}(t)$. The quantum dynamics can be obtained by averaging over sufficient number of trajectories

$$
\lambda_{\mu}^{i}(t) \approx \overline{\lambda_{\mu}^{i}(t)}=\sum_{\left\{a_{\mu}\right\}} p\left(\left\{a_{\mu}\right\}\right) \lambda_{\mu,\left\{a_{\mu}\right\}}^{i}(t) .
$$




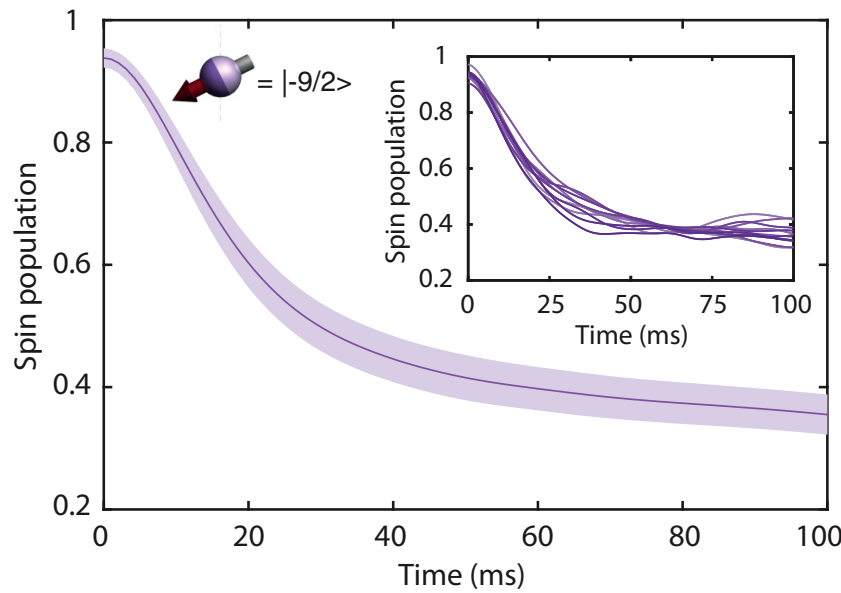

FIG. 7. Spin dynamics for $m_{F}^{0}=|-9 / 2\rangle$ as shown in Fig. 3 of the main text. The solid line represents the spin population and the shaded area denotes the standard deviation of individual trajectories which shows that the spread of the GDTWA trajectories does not grow large with time. The inset visualizes 10 typical trajectories obtained from the GDTWA simulation. Note that the different trajectories include both the quantum noise, which is essential to account for beyond-mean-field effects, and statistical noise, which averages out when sampling over enough trajectories.

This approach has been shown capable of capturing the buildup of quantum correlations [16,31]. In Fig. 7, we illustrate the typical trajectories and their spread in the spin dynamics obtained with GDWTA for $m_{F}^{0}=|-9 / 2\rangle$.

\section{APPENDIX H: INCORPORATING EXPERIMENTAL CONDITIONS IN NUMERICAL SIMULATION}

In our experiment, the lattice filling fraction is not unity when the spin dynamics takes place. The reduced filling fraction is due to two effects: the finite temperature and atom loss during the initial state preparation. To account for the effect of a finite temperature, we first obtain the density distribution before ramping up the lattice from a Fermi-Dirac distribution $n^{0}\left(\mathbf{r}_{i}\right)=\frac{1}{1+\exp \left\{\beta\left[\epsilon\left(\mathbf{r}_{i}\right)-\mu\right]\right\}}$, with parameters $\beta=1 / k_{B} T$ and $\mu$ matching the inferred experiment temperature $T$ and the total atom number $N_{0}=2.4 \times 10^{4}$. The function $\epsilon\left(\mathbf{r}_{i}\right)$ accounts for the weak external harmonic confinement. We compute the density distribution function after loading the atoms in the lattice, $n^{F}\left(\mathbf{r}_{i}\right)$, by simulating the lattice ramp which is possible since to an excellent approximation we can treat the system as noninteracting. Indeed, we neglect the dipolar interaction in the loading given that their magnitude is much lower than the Fermi energy of the gas. In the numerical simulation, we then sample the position of atoms $\mathbf{r}_{i}$ in the lattice according to a distribution $p\left(\mathbf{r}_{i}\right)=n^{F}\left(\mathbf{r}_{i}\right) / N_{0}$. In practice, to reduce computation cost we need to reduce the total atom number in our calculations and use a smaller lattice with fewer populated lattice sites. In this case, we reduce the number of lattice sites by a factor $\xi=\left(N_{\text {sim }} / N_{\exp }\right)^{1 / 3}$, where $N_{\text {sim(exp) }}$ are the number of atoms in the simulation (experiment), while keeping the lattice spacings the same as in experiment, $\left(d_{x}, d_{y}, d_{z}\right)=(272,266,544) \mathrm{nm}$. That is, for an initial lattice with $L_{x}$ sites along $x$ direction, in our

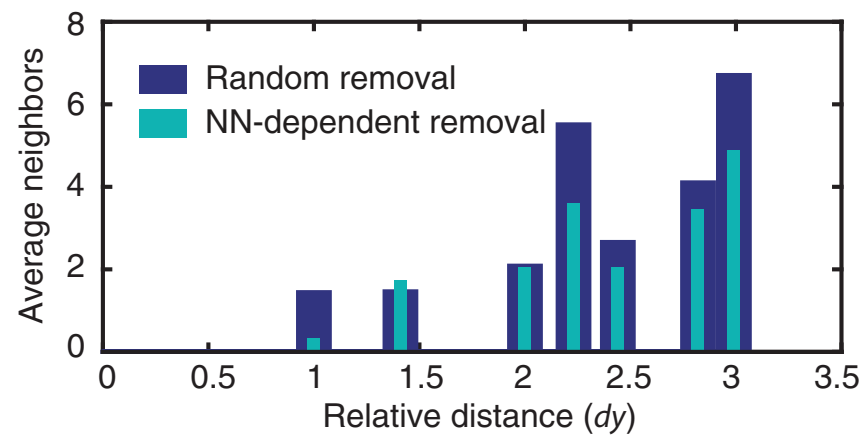

FIG. 8. Histogram showing the average number of atoms in distances normalized to the lattice direction along $y$ for random removal of atoms and for removal depending on the number of nearest neighbors ( $\mathrm{NN}$-dependent removal).

simulations there are $\xi L_{x}$ sites while the separation between two adjacent lattice sites is still $d_{x}$. We then sample the initial distribution of atoms in the lattice with $\tilde{p}\left(\tilde{\mathbf{r}}_{i}\right)=\xi^{3} p\left(\xi \tilde{\mathbf{r}}_{i}\right)$, which preserves the local density and is similar to sampling in a coarse-grained lattice. In our simulations, we chose $N_{\text {sim }} \gtrsim 350$ and checked that the convergence in $N_{\text {sim }}$ has been reached.

As discussed in Appendix B, a fraction of atoms is lost during the ramp up and down of the magnetic field before initializing the spin dynamics over the sample. While a rigorous treatment on how these losses modify the distribution is not currently accessible with our current experimental setup, we try to account for it in the simulation by preferentially removing those atoms with a probablity $\propto p\left(\mathbf{r}_{i}\right) N_{\mathrm{nn}}$, where $N_{\mathrm{nn}}$ is the number of nearest neighbors (separation $\leqslant d_{y}$ ), until $N=v(0) N_{0}$ atoms are left. According to experiment estimates, the filling fractions before the initialization of the spin dynamics are $v(0)=0.6-0.7$ (see Tables I and II). Figure 8 shows the histogram of neighbors in the resulting atom distribution. Such distribution effectively reduces the nearest-neighbor interactions and is found to give a better agreement with experiment.

Both the quadratic and linear shifts in the experiment are inhomogeneous across the lattice as discussed in Appendix E, and we include them in our numerical simulation as site-dependent terms $\delta_{i}\left(\hat{F}_{i}^{z}\right)^{2}$ and $B_{i} \hat{F}_{i}^{z}$, with $\delta_{i}=a\left|\mathbf{r}_{i}\right|^{2}$ and $B_{i}=b\left(x_{i}+y_{i}+z_{i}\right)$. Based on experimental estimation, we have chosen the values of $a$ and $b$ such that $\delta_{i}=h \times 1.6 \mathrm{~Hz}(h \times 0.7 \mathrm{~Hz})$ at 20 sites along $y$ away from the lattice center, and $B_{i}$ differs by $h \times 6 \mathrm{~Hz}(h \times 1.8 \mathrm{~Hz})$ between adjacent sites, for Fig. 2 and 3 (Fig. 4) in the simulation.

To illustrate the quantum spin dynamics under ideal conditions, in Fig. 2(b), we also show the results for atoms frozen in the lattice with unity filling fraction and zero temperature and all initialized in a single $m_{F}^{0}$ state. In the numerical simulation for this ideal case, a lattice size of $9 \times 9 \times 3$ is used, which accounts for the fact that the lattice spacing along $z$ is larger and thus the dipolar coupling decreases significantly within a few lattice sites. Even though numerical simulations cannot be performed for a lattice as large as that one in experiment, as 
shown in Ref. [16], for such a lattice configuration finite-size effects are negligible.

\section{APPENDIX I: SHORT-TIME POPULATION DYNAMICS}

Considering a fixed initial atomic distribution over the lattice, the population dynamics at early times can be derived via a perturbative short-time expansion

$$
\begin{aligned}
n_{m_{F}}(t) \equiv & \left\langle\hat{n}_{m_{F}}(t)\right\rangle=\left\langle\hat{n}_{m_{F}}\right\rangle+i\left\langle\left[\hat{H}, \hat{n}_{m_{F}}\right]\right\rangle t / \hbar \\
& -\left\langle\left[\hat{H},\left[\hat{H}, \hat{n}_{m_{F}}\right]\right]\right\rangle t^{2} / 2 \hbar^{2} \\
& -i\left\langle\left[\hat{H},\left[\hat{H},\left[\hat{H}, \hat{n}_{m_{F}}\right]\right]\right]\right\rangle t^{3} / 3 ! \hbar^{3} \\
& +\left\langle\left[\hat{H},\left[\hat{H},\left[\hat{H},\left[\hat{H}, \hat{n}_{m_{F}}\right]\right]\right]\right\rangle t^{4} / 4 ! \hbar^{4}+\mathcal{O}\left(t^{5}\right) .\right.
\end{aligned}
$$

Here the average $\langle\cdot\rangle$ is over the initial state, which is assumed to be a pure state, $\hat{n}_{m_{F}}=\left(\sum_{i} \mathcal{P}_{i}^{m_{F}}\right) / N$, where $\mathcal{P}_{i}^{m_{F}}=\left|m_{F}\right\rangle_{i i}\left\langle m_{F}\right|$ is the onsite projector for an atom at site $i$ in state $\left|m_{F}\right\rangle$ and $N$ denotes the total number of atoms. Note that here the sums are always carried out over the populated lattice sites in the initial lattice configuration. We obtain

$$
\begin{aligned}
V_{\mathrm{eff}}^{2}= & \frac{\gamma^{2}\left(m_{F}^{0}\right)}{8 N} \sum_{i, j \neq i} V_{i, j}^{2}, \\
\gamma\left(m_{F}^{0}\right)= & \sqrt{F(F+1)-m_{F}^{0}\left(m_{F}^{0}+1\right)} \\
& \times \sqrt{F(F+1)-m_{F}^{0}\left(m_{F}^{0}-1\right)},
\end{aligned}
$$

where $n_{m_{F}^{0}}$ denotes the population on the selected target state. To obtain Eq. (I2), we have assumed that initially most of the population is in this target state, i.e., $n_{m_{F}^{0}}(0) \sim 1$. In the experiment, this assumption is always satisfied and therefore Eq. (I4) is expected to reproduce well the short-time dynamics.

The dependence of $\gamma\left(m_{F}^{0}\right)$ on the initial state $m_{F}^{0}$ is a consequence of the dependence of dipolar exchange processes on the spin coherences, i.e., $\left|\left\langle i: m_{F}^{0}+1, j: m_{F}^{0}-1\left|\hat{F}_{i}^{+} \hat{F}_{j}^{-}\right| i: m_{F}^{0}, j: m_{F}^{0}\right\rangle\right|$. Therefore the smaller the value $\left|m_{F}^{0}\right|$ of the initial populated states, the faster the early-time dynamics. Notably, to order $t^{2}$ the initial dynamics is independent of quadratic shifts and external magnetic field gradients. This is because both of their corresponding Hamiltonians commute with the spin population operator $\hat{n}_{m_{F}}$. From this simple perturbative treatment one learns that by preparing different initial states with different $m_{F}^{0}$, the decay rates of the short-time population dynamics provide information of $V_{\text {eff }}$ and thus of the underlying dipolar couplings. As discussed in Appendices $\mathrm{B}$ and $\mathrm{H}$, the lattice filling fraction is not unity and the initial atomic density distribution in the lattice may vary from shot to shot. To account for this effect, we perform a statistical average of Eq. (I3) calculated for each lattice configuration generated with the procedure in Appendix $\mathrm{H}$ to obtain the theoretical values in Fig. 3(g) and Fig. 4(b).

It is important here to compare the predictions obtained from a simple mean-field analysis. In contrast to Eq. (I4), neglecting quantum correlations yields

$$
\begin{aligned}
& n_{m_{F}^{0}}^{\text {Mean-Field }}(t) \\
& \quad=n_{m_{F}^{0}}(0)\left\{1-n_{m_{F}^{0}}(0)\left[1-n_{m_{F}^{0}}(0)\right] \frac{V_{\text {eff }}^{2}}{\hbar^{2}} t^{2}+\mathcal{O}\left(t^{4}\right)\right\} .
\end{aligned}
$$

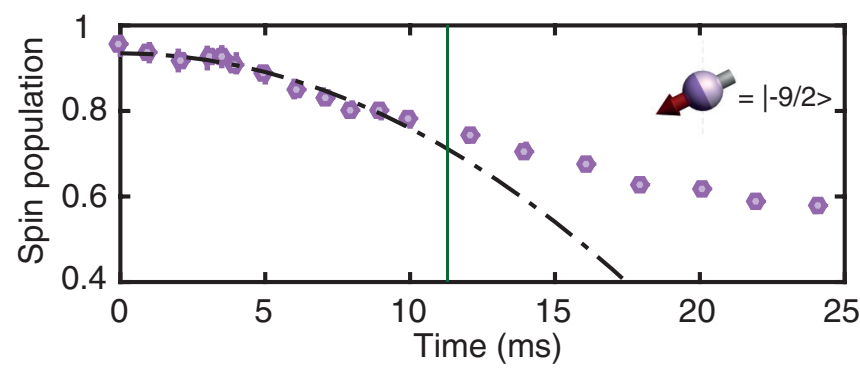

FIG. 9. The dotted-dashed line exemplary shows the fit of Eq. (I2) to the experimental data to extract $V_{\text {eff }}$ for $m_{F}^{0}=|-9 / 2\rangle$. The solid green line indicates the time $t_{\text {fit }}$ up to which the fit is performed.

At the mean-field level, therefore, if initially the atoms are prepared such that $n_{m_{r}^{0}}(0)=1$, then there is no population dynamics. This is in stark contrast to the quantum systems where dynamics is enabled by quantum fluctuations. To extract $V_{\text {eff }}$ from our experimental data and to compare it to the theoretical simulations we fit the initial dynamics with Eq. (I4). We define the timescale for the fitting via $t_{\text {fit }}<0.5 \frac{\hbar}{V_{\text {eff }}}$, which corresponds to the timescale on which each atom did on average half a spin flip. We note that on this timescale the time evolution starts already to deviate from the short-time expansion [Eq. (2)], leading to a systematic downshift of the experimentally fitted $V_{\text {eff }}$; see Fig. 4(b). However, a minimum timescale has to be chosen to ensure that the fit is performed using a large-enough number of data points. Figure 9 shows exemplary the fit to the experimental data for $m_{F}^{0}=|-9 / 2\rangle$. In Fig. 10 we show for completeness the experimental data as well as the theory calculations for all spin states with the rescaled time axis [see Fig. 3(h)].

\section{APPENDIX J: EFFECT FROM TUNNELING PROCESSES}

In our experiment, the tunneling rate is small but finite. To understand its possible effect on the measured spin dynamics, we recall that our system can be described by a

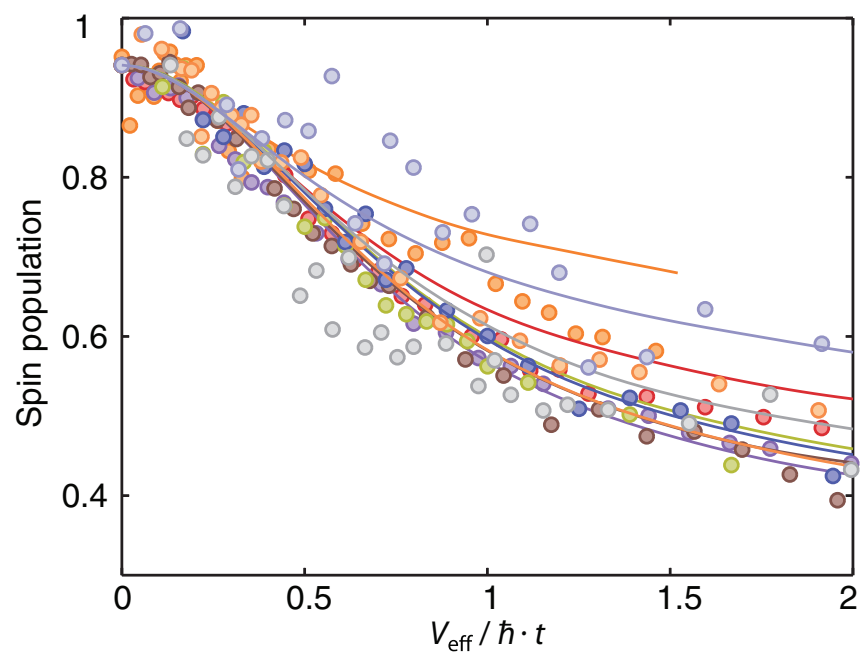

FIG. 10. Expansion of Fig. 3(h) showing all measured spin states and the corresponding theory predictions. 
Fermi-Hubbard Hamiltonian:

$$
\hat{H}_{\mathrm{FH}}=\hat{H}_{\mathrm{tun}}+\hat{H}_{\mathrm{inho}}+\hat{H}_{V_{d d}}+\hat{H}_{U_{d d}}+\hat{H}_{s}
$$

with

$$
\begin{aligned}
\hat{H}_{\text {tun }}= & -\sum_{\langle\mathbf{i}, \mathbf{j}\rangle, m}\left(J_{\mathbf{i}, \mathbf{j}} \hat{f}_{\mathbf{i} m}^{\dagger} \hat{f}_{\mathbf{j} m}+\text { H.c. }\right), \\
\hat{H}_{\text {inho }}= & \sum_{\mathbf{i}} \sum_{m, n} \delta_{\mathbf{i}} \hat{f}_{\mathbf{i} m}^{\dagger}\left(F_{m n}^{z}\right)^{2} \hat{f}_{\mathbf{i} n}+\sum_{\mathbf{i}} \sum_{m, n} B_{\mathbf{i}} \hat{f}_{\mathbf{i} m}^{\dagger} \hat{F}_{m n}^{z} \hat{f}_{\mathbf{i} n}, \\
\hat{H}_{V_{d d}}= & \frac{1}{2} \sum_{\mathbf{i} \neq \mathbf{j}} \sum_{m, n, k, l} V_{\mathbf{i}, \mathbf{j}}\left[F_{m n}^{z} F_{k l}^{z}-\frac{1}{4}\left(F_{m n}^{+} F_{k l}^{-}+F_{m n}^{-} F_{k l}^{+}\right)\right] \\
& \times \hat{f}_{\mathbf{i} m}^{\dagger} \hat{f}_{\mathbf{i} k}^{\dagger} \hat{f}_{\mathbf{j} l} \hat{f}_{\mathbf{j} n}, \\
\hat{H}_{U_{d d}}= & \frac{1}{2} U_{d d} \sum_{\mathbf{i}} \sum_{m, n, k, l}\left[F_{m n}^{z} F_{k l}^{z}-\frac{1}{4}\left(F_{m n}^{+} F_{k l}^{-}+F_{m n}^{-} F_{k l}^{+}\right)\right] \\
& \times \hat{f}_{\mathbf{i} m}^{\dagger} \hat{f}_{\mathbf{i} k}^{\dagger} \hat{f}_{\mathbf{i} l} \hat{f}_{\mathbf{i} n},
\end{aligned}
$$

$$
\hat{H}_{s}=\frac{1}{2} \sum_{\mathbf{i}} \sum_{m, n, k, l} U_{m n k l} \hat{f}_{\mathbf{i} m}^{\dagger} \hat{f}_{\mathbf{i} k}^{\dagger} \hat{f_{\mathbf{i} l}} \hat{f_{\mathbf{i}} n},
$$

where $\hat{f}_{\mathbf{i} m}\left(\hat{f}_{\mathbf{i} m}^{\dagger}\right)$ annihilates (creates) a fermion of spin state $m$ on site $\mathbf{i}=\left\{i_{x}, i_{y}, i_{z}\right\}$, and $F_{m n}^{z,+,-}$ are the matrix elements of the corresponding spin-19/2 angular momentum operators. The first term describes the single-particle tunneling between adjacent sites, with the tunneling rate $J$ calculated from the integral over the lowest band Wannier functions:

$$
J_{\mathbf{i}, \mathbf{j}}=-\int d^{3} \mathbf{r} \phi_{\mathbf{i}}^{*}(\mathbf{r})\left[-\frac{\hbar^{2} \nabla^{2}}{2 M}+V_{\text {latt }}(\mathbf{r})\right] \phi_{\mathbf{j}}(\mathbf{r}),
$$

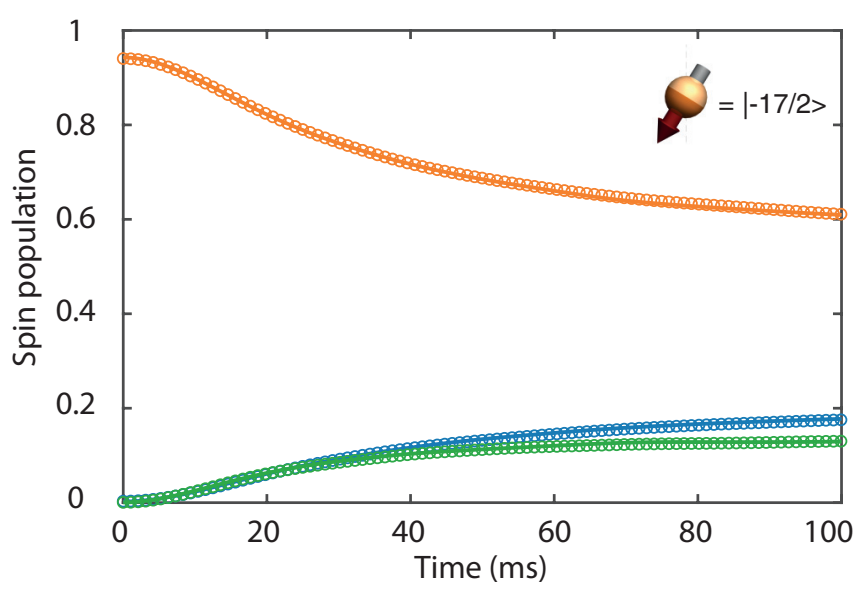

FIG. 11. Comparison between spin dynamics obtained with the reduced spin model Eq. (F1) (solid lines) and the Hamiltonian Eq. (J12) accounting for superexchange interactions (circles) for initial state $m_{F}^{0}=|-17 / 2\rangle$ and typical experimental conditions. Results are shown for the three most populated spin states, $m_{F}^{0}, m_{F}^{0} \pm 1$. For the on-site interaction strengths, we adopt for $a_{18}$ the value measured in Ref. [29] and assume the rest $a_{S}$ 's are randomly distributed within $\pm 40 \%$ with respect to $a_{18}$. For identical $a_{S}$ and absent of $U_{d d}$ the superexchange Hamiltonian has a $\mathrm{SU}(2 F+1)$ symmetry that does not change spin population. We expect that the reasonable variation between $a_{S}$ 's of ${ }^{167} \mathrm{Er}$ atoms does not exceed $\pm 40 \%$. where $M$ denotes the atomic mass and $V_{\text {latt }}$ is the external lattice potential. The second term $\hat{H}_{\text {inho includes the site- }}$ dependent quadratic and linear shifts, respectively. In the experiment, initially all atoms are prepared in the same spin state. At the achieved initial temperature at which atoms are restricted to occupy the lowest lattice band, Fermi statistics prevents more than one atom per lattice site. Dipolar exchange processes can change the atomic internal spin states, allowing tunneling to happen. When two Er atoms occupy the same lattice site they interact with each other via both a contact interaction governed by the Hamiltonian $\hat{H}_{s}$ and an on-site dipolar interaction governed by $\hat{H}_{U_{d d}}$, which are the last two terms in Eq. (J1). The strength of on-site dipolar interaction can be obtained from

$$
U_{d d}=\frac{\mu_{0}\left(\mu_{B} g_{F}\right)^{2}}{4 \pi} \int d^{3} \mathbf{r} d^{3} \mathbf{r}^{\prime} \frac{1-3 \cos ^{2} \theta}{\left|\mathbf{r}-\mathbf{r}^{\prime}\right|^{3}}|\phi(\mathbf{r})|^{2}\left|\phi\left(\mathbf{r}^{\prime}\right)\right|^{2},
$$

with the integral over Wannier functions at the same lattice site, $\phi(\mathbf{r})$. For dipoles oriented along $z$, (i.e., $\Theta=0$, see Fig. 1), the experimental lattice geometry leads to an attractive net $U_{d d}$. For $s$-wave scattering, the interaction kernel in $\hat{H}_{s}$ can

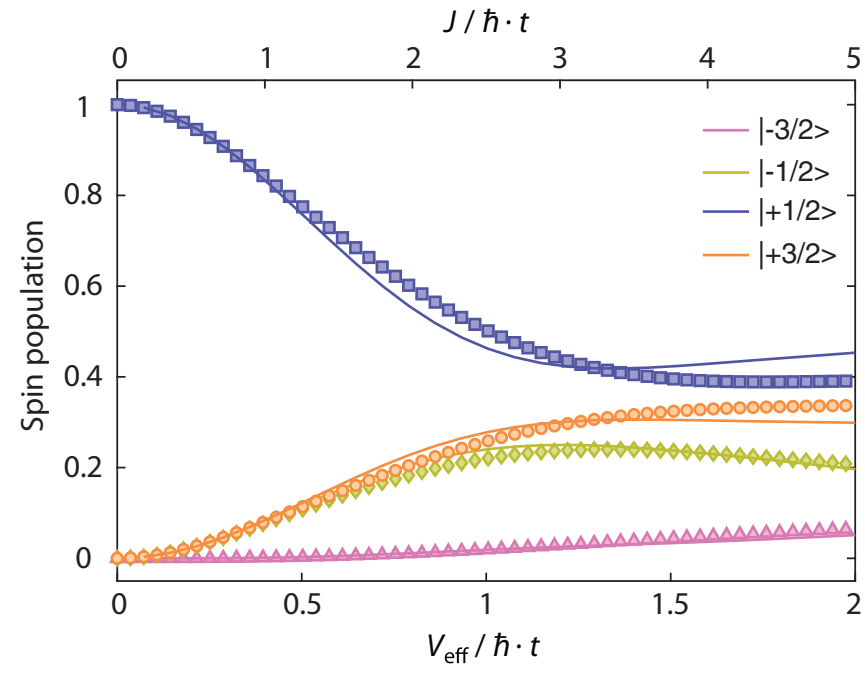

FIG. 12. Spin dynamics from the Fermi-Hubbard model with $F=3 / 2$ (symbols). A one-dimensional system with eight sites is used, with uniform nearest-neighbor tunneling rate $J_{\mathbf{i}, \mathbf{j}}=J$. To reduce finite-size effect, a periodic boundary condition is adopted. We assume an on-site contact interaction $U_{s}$ with $\mathrm{SU}(2 F+1)$ symmetry and strength $U_{s} / J=200$, approximately corresponding to the value measured in Ref. [29] and $J / \hbar=10 \mathrm{~Hz}$. Since $U_{s}$ is much stronger than the on-site dipolar interaction for the erbium experiment [29], we do not include $U_{d d}$ in these calculations. We have also neglected external inhomogeneous fields [Eq. (J3)] in the model. Initially five sites are occupied with atoms all prepared in the $m_{F}^{0}=1 / 2$ state. The spin dynamics is calculated using exact diagonalization and averaged over all possible initial distribution of empty sites. To provide a reference for the $F=19 / 2$ erbium case, we have focused on a timescale similar to the one probed in experiment, with $V_{\text {eff }}$ defined as in the main text and setting $F=3 / 2$ in the expression for $\gamma\left(m_{F}^{0}\right)$. As a comparison, the spin dynamics obtained with the frozen spin model [Eq. (F1)] with the same lattice configuration is also plotted with solid lines. 
be generally rewritten in terms of

$$
\begin{aligned}
U_{m n k l}= & \sum_{S=0,2, \ldots}^{2 F-1} c_{S}\left(a_{S}\right) \sum_{m_{S}=-S}^{S}\left(\left\langle F, m ; F, k \mid S, M_{S}\right\rangle\right. \\
& \left.\times\left\langle S, M_{S} \mid F, n ; F, l\right\rangle\right),
\end{aligned}
$$

where $S$ is the total angular momentum of the colliding particles and $M_{S}$ its projection along the quantization axis. The subscripts $m, n, k, l$ run from $-19 / 2$ to $19 / 2$. Odd values of $F$ are forbidden for $s$-wave collision. Here

$$
c_{S}\left(a_{S}\right)=\frac{4 \pi \hbar^{2} a_{S}}{M} \int d^{3} \mathbf{r}|\phi(\mathbf{r})|^{4}
$$

characterizing the scattering in the total spin $S$ channel and $a_{S}$ is the corresponding background scattering length. For ${ }^{167} \mathrm{Er}$, with $F=19 / 2$ there are in principle 10 different scattering lengths. Most of the $a_{S}$ remain unknown, except the one between $m=-19 / 2$ and $n=-17 / 2$, which was measured to be $\sim 91(8) a_{0}$ with $a_{0}$ the Bohr radius [29]. Given the complex molecular potential of Er atoms we expect not significant variations between them.

In the deep lattice regime, the on-site interactions are of the order of $\mathrm{kHz}$ [29] and therefore much stronger than the tunneling rate $\sim 10 \mathrm{~Hz}$. As a consequence, tunneling processes between two adjacent occupied lattice sites are energetically forbidden and they contribute only as second-order virtual processes also known as superexchange interactions. They take the form

$$
\hat{H}_{\mathrm{ex}}^{\langle\mathbf{i}, \mathbf{j}\rangle}=-\sum_{\gamma} \frac{\left\langle\alpha\left|\hat{H}_{\mathrm{tun}}\right| \gamma\right\rangle\left\langle\gamma\left|\hat{H}_{\mathrm{tun}}\right| \beta\right\rangle}{\left\langle\gamma\left|\hat{H}_{s}\right| \gamma\right\rangle+\left\langle\gamma\left|\hat{H}_{U_{d d}}\right| \gamma\right\rangle}|\alpha\rangle\langle\beta|,
$$

where $\alpha, \beta$ denote a basis set spanned by states where two adjacent sites $\mathbf{i}, \mathbf{j}$ are each occupied by one atom and $\gamma$ denotes the set of states where two atoms occupy the same site and are diagonal in both the $s$-wave and on-site dipolar interaction Hamiltonians, $\hat{H}_{s}, \hat{H}_{U_{d d}}$. The superscript $\langle\mathbf{i}, \mathbf{j}\rangle$ emphasizes that the superexchange interaction only occurs between nearestneighbor sites.

After accounting for all these interactions, we can obtain an effective spin Hamiltonian by projecting to the physical subspace with at most one atom per site and also neglecting tunneling processes to empty sites, which anyway do not modify the magnetic character of the system:

$$
\hat{H}^{\prime}=\hat{H}+\sum_{\langle\mathbf{i}, \mathbf{j}\rangle} \hat{H}_{\mathrm{ex}}^{\langle\mathbf{i}, \mathbf{j}\rangle},
$$

where $\hat{H}$ is the spin Hamiltonian given in Appendix F.

To get a basic idea of the resulting superexchange interactions, we first provide as an example the case of $F=3 / 2$, for which the Hamiltonian obtained using the above procedure for two adjacent sites is

$$
\hat{H}_{\mathrm{ex}}^{(\mathbf{i}, \mathbf{j}\rangle}=\left(\begin{array}{cccccccccccccccc}
0 & 0 & 0 & 0 & 0 & 0 & 0 & 0 & 0 & 0 & 0 & 0 & 0 & 0 & 0 & 0 \\
0 & -a & 0 & 0 & a & 0 & 0 & 0 & 0 & 0 & 0 & 0 & 0 & 0 & 0 & 0 \\
0 & 0 & b & 0 & 0 & 0 & 0 & 0 & -b & 0 & 0 & 0 & 0 & 0 & 0 & 0 \\
0 & 0 & 0 & c & 0 & 0 & d & 0 & 0 & -d & 0 & 0 & -c & 0 & 0 & 0 \\
0 & a & 0 & 0 & -a & 0 & 0 & 0 & 0 & 0 & 0 & 0 & 0 & 0 & 0 & 0 \\
0 & 0 & 0 & 0 & 0 & 0 & 0 & 0 & 0 & 0 & 0 & 0 & 0 & 0 & 0 & 0 \\
0 & 0 & 0 & d & 0 & 0 & e & 0 & 0 & -e & 0 & 0 & -d & 0 & 0 & 0 \\
0 & 0 & 0 & 0 & 0 & 0 & 0 & b & 0 & 0 & 0 & 0 & 0 & -b & 0 & 0 \\
0 & 0 & -b & 0 & 0 & 0 & 0 & 0 & b & 0 & 0 & 0 & 0 & 0 & 0 & 0 \\
0 & 0 & 0 & -d & 0 & 0 & -e & 0 & 0 & e & 0 & 0 & d & 0 & 0 & 0 \\
0 & 0 & 0 & 0 & 0 & 0 & 0 & 0 & 0 & 0 & 0 & 0 & 0 & 0 & 0 & 0 \\
0 & 0 & 0 & 0 & 0 & 0 & 0 & 0 & 0 & 0 & 0 & -a & 0 & 0 & a & 0 \\
0 & 0 & 0 & -c & 0 & 0 & -d & 0 & 0 & d & 0 & 0 & c & 0 & 0 & 0 \\
0 & 0 & 0 & 0 & 0 & 0 & 0 & -b & 0 & 0 & 0 & 0 & 0 & b & 0 & 0 \\
0 & 0 & 0 & 0 & 0 & 0 & 0 & 0 & 0 & 0 & 0 & a & 0 & 0 & -a & 0 \\
0 & 0 & 0 & 0 & 0 & 0 & 0 & 0 & 0 & 0 & 0 & 0 & 0 & 0 & 0 & 0
\end{array}\right),
$$

where

$$
\begin{aligned}
& a=\frac{4 J^{2}}{3 U_{d d}+2 U_{s}}, \\
& b=\frac{8 J^{2}}{3 U_{d d}-4 U_{s}}, \\
& c=\frac{2 J^{2}\left[3 U_{d d}+2 U_{s}\left(2+x_{0}\right)\right]}{9 U_{d d}^{2}+6 U_{d d} U_{s}\left(1+x_{0}\right)-4 U_{s}^{2}\left(1+x_{0}\right)},
\end{aligned}
$$

$$
\begin{aligned}
& d=\frac{2 J^{2}\left(3 U_{d d}+2 U_{s} x_{0}\right)}{9 U_{d d}^{2}+6 U_{d d} U_{s}\left(1+x_{0}\right)-4 U_{s}^{2}\left(1+x_{0}\right)}, \\
& e=\frac{2 J^{2}\left[-9 U_{d d}+2 U_{s}\left(2+x_{0}\right)\right]}{9 U_{d d}^{2}+6 U_{d d} U_{s}\left(1+x_{0}\right)-4 U_{s}^{2}\left(1+x_{0}\right)},
\end{aligned}
$$

$J$ is the nearest-neighbor tunneling rate,

$$
U_{s}=\frac{4 \pi \hbar^{2} a_{2}}{M} \int d \mathbf{r}|\phi(\mathbf{r})|^{4},
$$


and $x_{0}=a_{0} / a_{2}-1$ denotes the fractional difference between the scattering lengths of the two channels with total spin 0 and 2. With this explicit form of the superexchange Hamiltonian, we can find the short-time dynamics for atoms initialized in the same state $m_{F}^{0}=|-1 / 2\rangle$ :

$$
n_{-1 / 2}=1-\frac{3 t^{2}}{4} \sum_{i, j \neq i} V_{i, j}^{2}+\mathcal{O}\left(t^{4}\right),
$$

which indicates that superexchange interactions do not affect the initial dynamics, as one would expect from Fermi statistics.

Although for the case of Er atoms with $F=19 / 2$ we do not have a simple analytical expression for the final Hamiltonian Eq. (J12) we can obtain it numerically. Moreover, since for Er most $a_{S}$ are unknown we assume random values for $a_{S}$ and solve the ensuing dynamics with the GDTWA approach.
In Fig. 11, we plot the result for a typical initial state $m_{F}^{0}=$ $|-17 / 2\rangle$, which shows that the addition of superexchange interactions hardly affects the measured spin dynamics during the relevant timescale.

To further analyze the role of tunneling during the dynamics and account for the presence of initially unoccupied sites, we calculate the spin dynamics from the FermiHubbard model Eq. (J1). In Fig. 12, we plot the results obtained for a small-size system using exact diagonalization and assuming $\sim 40 \%$ holes at $t=0$. At short times $J / \hbar \cdot t \sim 1$, tunneling effect is negligible. At longer times, tunneling interplays with interaction and Fermi statistics and modifies the quantum dynamics. Nevertheless, these results suggest that such modification is not significant for the most relevant timescales probed in this work. Therefore, for the comparison with experimental observations in this work, we use the reduced spin model Eq. (F1) and neglect tunneling altogether.
[1] I. Bloch, Quantum coherence and entanglement with ultracold atoms in optical lattices, Nature 453, 1016 (2008).

[2] C. Gross and I. Bloch, Quantum simulations with ultracold atoms in optical lattices, Science 357, 995 (2017).

[3] I. Bloch, J. Dalibard, and W. Zwerger, Many-body physics with ultracold gases, Rev. Mod. Phys. 80, 885 (2008).

[4] D. Greif, T. Uehlinger, G. Jotzu, L. Tarruell, and T. Esslinger, Short-range quantum magnetism of ultracold fermions in an optical lattice, Science 340, 1307 (2013).

[5] J. Zeiher, J.-Y. Choi, A. Rubio-Abadal, T. Pohl, R. van Bijnen, I. Bloch, and C. Gross, Coherent Many-Body Spin Dynamics in a Long-Range Interacting Ising Chain, Phys. Rev. X 7, 041063 (2017).

[6] H. Bernien, S. Schwartz, A. Keesling, H. Levine, A. Omran, H. Pichler, S. Choi, A. S. Zibrov, M. Endres, M. Greiner, V. Vuletić, and M. D. Lukin, Probing many-body dynamics on a 51-atom quantum simulator, Nature 551, 579 (2017).

[7] D. Barredo, V. Lienhard, S. de Leseleuc, T. Lahaye, and A. Browaeys, Synthetic three-dimensional atomic structures assembled atom by atom, Nature 561, 79 (2018).

[8] E. Guardado-Sanchez, P. T. Brown, D. Mitra, T. Devakul, D. A. Huse, P. Schauß, and W. S. Bakr, Probing the Quench Dynamics of Antiferromagnetic Correlations in a 2d Quantum Ising Spin System, Phys. Rev. X 8, 021069 (2018).

[9] B. Neyenhuis, J. Zhang, P. W. Hess, J. Smith, A. C. Lee, P. Richerme, Z.-X. Gong, A. V. Gorshkov, and C. Monroe, Observation of prethermalization in long-range interacting spin chains, Sci. Adv. 3, e1700672 (2017).

[10] R. Blatt and C. F. Roos, Quantum simulations with trapped ions, Nat. Phys. 8, 277 (2012).

[11] J. W. Britton, B. C. Sawyer, A. C. Keith, C. C.. Wang, J. K. Freericks, H. Uys, M. J. Biercuk, and J. J. Bollinger, Engineered two-dimensional ising interactions in a trapped-ion quantum simulator with hundreds of spins, Nature 484, 489 (2012).

[12] B. Yan, S. A. Moses, B. Gadway, J. P. Covey, K. R. A. Hazzard, A. M. Rey, D. S. Jin, and J. Ye, Observation of dipolar spinexchange interactions with lattice-confined polar molecules, Nature 501, 521 (2013).
[13] K. R. A. Hazzard, B. Gadway, M. Foss-Feig, B. Yan, S. A. Moses, J. P. Covey, N. Y. Yao, M. D. Lukin, J. Ye, D. S. Jin, and A. M. Rey, Many-Body Dynamics of Dipolar Molecules in an Optical Lattice, Phys. Rev. Lett. 113, 195302 (2014).

[14] A. de Paz, A. Sharma, A. Chotia, E. Maréchal, J. H. Huckans, P. Pedri, L. Santos, O. Gorceix, L. Vernac, and B. Laburthe-Tolra, Nonequilibrium Quantum Magnetism in a Dipolar Lattice Gas, Phys. Rev. Lett. 111, 185305 (2013).

[15] A. de Paz, P. Pedri, A. Sharma, M. Efremov, B. Naylor, O. Gorceix, E. Maréchal, L. Vernac, and B. Laburthe-Tolra, Probing spin dynamics from the Mott insulating to the superfluid regime in a dipolar lattice gas, Phys. Rev. A 93, 021603(R) (2016).

[16] S. Lepoutre, J. Schachenmayer, L. Gabardos, B. Zhu, B. Naylor, E. Maréchal, O. Gorceix, A. M. Rey, L. Vernac, and B. Laburthe-Tolra, Out-of-equilibrium quantum magnetism and thermalization in a spin-3 many-body dipolar lattice system, Nat. Commun. 10, 1714 (2019).

[17] D. M. Stamper-Kurn and M. Ueda, Spinor bose gases: Symmetries, magnetism, and quantum dynamics, Rev. Mod. Phys. 85, 1191 (2013).

[18] J. H. Becher, S. Baier, K. Aikawa, M. Lepers, J.-F. Wyart, O. Dulieu, and F. Ferlaino, Anisotropic polarizability of erbium atoms, Phys. Rev. A 97, 012509 (2018).

[19] We note that bosonic $\mathrm{Cr}$ [16] and bosonic Er have zero quadratic Zeeman shift because $I=0$.

[20] B. Zhu, A. M. Rey, and J. Schachenmayer, A generalized phase space approach for solving quantum spin dynamics, New J. Phys. 21, 082001 (2019).

[21] M. Mancini, G. Pagano, G. Cappellini, L. Livi, M. Rider, J. Catani, C. Sias, P. Zoller, M. Inguscio, M. Dalmonte, and L. Fallani, Observation of chiral edge states with neutral fermions in synthetic hall ribbons, Science 349, 1510 (2015).

[22] B. K. Stuhl, H.-I. Lu, L. M. Aycock, D. Genkina, and I. B. Spielman, Visualizing edge states with an atomic bose gas in the quantum hall regime, Science 349, 1514 (2015).

[23] B. Sundar, B. Gadway, and K. R. A. Hazzard, Synthetic dimensions in ultracold polar molecules, Sci. Rep. 8, 3422 (2018). 
[24] B. P. Lanyon, M. Barbieri, M. P. Almeida, T. Jennewein, T. C. Ralph, K. J. Resch, G. J. Pryde, J. L. O’Brien, A. Gilchrist, and A. G. White, Simplifying quantum logic using higherdimensional hilbert spaces, Nat. Phys. 5, 134 (2008).

[25] E. Brion, K. Mølmer, and M. Saffman, Quantum Computing with Collective Ensembles of Multilevel Systems, Phys. Rev. Lett. 99, 260501 (2007).

[26] M. Kues, C. Reimer, P. Roztocki, L. R. Cortés, S. Sciara, B. Wetzel, Y. Zhang, A. Cino, S. T. Chu, B. E. Little, D. J. Moss, L. Caspani, J. Azaña, and R. Morandotti, On-chip generation of high-dimensional entangled quantum states and their coherent control, Nature 546, 622 (2017).

[27] A. Auerbach, Interacting Electrons and Quantum Magnetism (Springer-Verlag, New York, 1994).

[28] O. Dutta, M. Gajda, P. Hauke, M. Lewenstein, D.-S. Lühmann, B. A. Malomed, T. Sowiński, and J. Zakrzewski, Non-standard hubbard models in optical lattices: A review, Rep. Prog. Phys. 78, 066001 (2015).

[29] S. Baier, D. Petter, J. H. Becher, A. Patscheider, G. Natale, L. Chomaz, M. J. Mark, and F. Ferlaino, Realization of a Strongly Interacting Fermi Gas of Dipolar Atoms, Phys. Rev. Lett. 121, 093602 (2018).

[30] J. Schachenmayer, A. Pikovski, and A. M. Rey, Dynamics of correlations in two-dimensional quantum spin models with long-range interactions: A phase-space monte-carlo study, New J. Phys. 17, 065009 (2015).

[31] J. Schachenmayer, A. Pikovski, and A. M. Rey, ManyBody Quantum Spin Dynamics with Monte Carlo Trajectories on a Discrete Phase Space, Phys. Rev. X 5, 011022 (2015).
[32] A. Polkovnikov, Phase space representation of quantum dynamics, Ann. Phys. 325, 1790 (2010)

[33] We note that dissipationless dynamics is the most justified on this timescale as losses stay below $14 \%$.

[34] A. V. Gorshkov, S. R. Manmana, G. Chen, E. Demler, M. D. Lukin, and A. M. Rey, Quantum magnetism with polar alkalimetal dimers, Phys. Rev. A 84, 033619 (2011).

[35] C. Weitenberg, M. Endres, J. F. Sherson, M. Cheneau, P. Schauß, T. Fukuhara, I. Bloch, and S. Kuhr, Single-spin addressing in an atomic Mott insulator, Nature 471, 319 (2011).

[36] T. Fukuhara, A. Kantian, M. Endres, M. Cheneau, P. Schauß, S. Hild, D. Bellem, U. Schollwöck, T. Giamarchi, C. Gross, I. Bloch, and S. Kuhr, Quantum dynamics of a mobile spin impurity, Nat. Phys. 9, 235 (2013).

[37] R. Zupancic, P. M. Preiss, R. Ma, A Lukin, M. E. Tai, M. Rispoli, R. Islam, and M. Greiner, Ultra-precise holographic beam shaping for microscopic quantum control, Opt. Express 24, 13881 (2016).

[38] H. Y. Ban, M. Jacka, J. L. Hanssen, J. Reader, and J. J. McClelland, Laser cooling transitions in atomic erbium, Opt. Express 13, 3185 (2005).

[39] K. Aikawa, A. Frisch, M. Mark, S. Baier, R. Grimm, and F. Ferlaino, Reaching Fermi Degeneracy Via Universal Dipolar Scattering, Phys. Rev. Lett. 112, 010404 (2014).

[40] S. Baier, M. J. Mark, low D. Petter, K. Aikawa, L. Chomaz, Z. Cai, M. Baranov, P. Zoller, and F. Ferlaino, Extended bosehubbard models with ultracold magnetic atoms, Science 352, 201 (2016).

[41] R. A. Bertlmann and P. Krammer, Bloch vectors for qudits, J. Phys. A: Math. Theor. 41, 235303 (2008). 